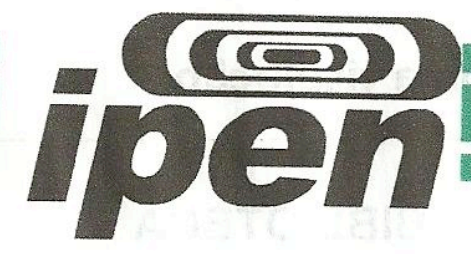

AUTARQUIA ASSOCIADA À UNIVERSIDADE DE SÃO PAULO

Desenvolvimento de cálculo de unidades monitoras para IMRT

Adriana Aparecida Flosi

Dissertação apresentada como parte dos requisitos para obtenção do Grau de Mestre em Ciências na Área de Tecnologia Nuclear - Aplicações

Orientadora:

Profa. Dra. Laura Natal Rodrigues 
Dedico este trabalho àqueles que eu amo.

$\mathrm{E}$ a todos que me ajudaram a chegar até aqui. 


\section{AGRADECIMENTOS}

À minha orientadora, Dra. Laura Natal Rodrigues, por ter me dado a oportunidade de realizar este trabalho.

À minha família Luiz, Lucimara, Ronaldo e, especialmente, Lucinéia, minha mãe.

Aos meus amigos da graduação: Juliano, Vanks, Carol e Amábile, pela grande e duradoura amizade.

À física Juliana pela ajuda com a aquisição dos dados, pelas valiosas discussões e pelo ombro amigo.

Aos amigos de trabalho Marcel, Joyce, Irene e Dayanne, pela confiança e incentivo nas horas árduas.

Aos físicos do departamento de radioterapia do Hospital A. C. Camargo, por terem permitido o uso dos aparelhos e materiais necessários para o desenvolvimento do trabalho.

Aos professores do IPEN que sempre estiveram à disposição. 


\title{
DESENVOLVIMENTO DE CÁLCULO DE UNIDADES MONITORAS PARA IMRT
}

\author{
Adriana Aparecida Flosi
}

\section{RESUMO}

A verificação de forma independente do cálculo de dose e de unidades monitoras num plano de tratamento de IMRT é um passo importante nos procedimentos de garantia de qualidade da técnica em questão. Atualmente este verificação é baseada apenas em medidas experimentais demoradas e trabalhosas.

Neste trabalho foi desenvolvido uma metodologia de cálculo de unidades monitoras de forma independente como uma nova ferramenta para garantir a qualidade e exatidão dos tratamentos de IMRT. Os valores encontrados se aproximam bastante dos valores calculados pelo sistema de planejamento utilizado, de forma que o algoritmo de cálculo desenvolvido apresentou uma concordância dentro de $\pm 1,8 \%$ para uma geometria simples.

Após diversos testes e com os níveis de ação devidamente estabelecidos, a verificação independente da unidade monitora para planos de tratamento de IMRT se tornará uma ferramenta efetiva e eficiente no controle de qualidade que ajuda a identificar e reduzir possíveis erros de tratamento em radioterapia.

Como contribuição original deste trabalho, assegura-se aos serviços de Radioterapia a utilização da metodologia desenvolvida como ferramenta de controle de qualidade em tratamentos com IMRT. Em especial aos serviços que não dispõem de recursos econômicos para adquirirem softwares comercialmente disponíveis para o cálculo independente da unidade monitora. 
DEVELOPEMENT OF MONITOR UNIT CALCULATION IN IMRT

Adriana Aparecida Flosi

\begin{abstract}
Independent verification of dose calculations and monitor units settings of IMRT treatment plans is an important step in the quality assurance procedure for IMRT technique. At present, the verification is mainly based on experimental measurements, which are time consuming and laborious.

In this work an independent methodology of monitor units calculation was developed as a new tool for IMRT treatments quality and precision assurance. The values found are near those calculated by the treatment planning system used, in a manner that the calculation algorithm demonstrated $\pm 1,8 \%$ concordance in a simple geometry with the system.

After several tests and the levels of action well established, the independent monitor units verification for IMRT treatment plans will become an effective and efficient tool in quality assurance, helping identification and the reduction of possible mistakes in radiotherapy treatments.

To radiotherapy services is assured the use of the developed methodology as a tool of quality control in IMRT treatments as an original contribution of this work, specially those that do not dispose financial resources to acquire commercially available independent monitor unit calculus software.
\end{abstract}




\section{SUMÁRIO}

Página

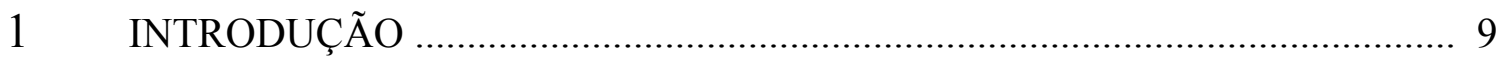

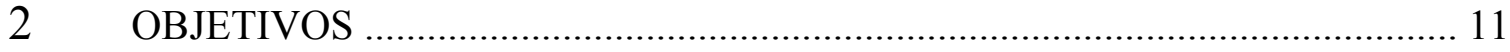

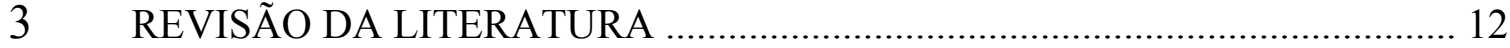

3.1 Comparação entre radioterapia conformacional 3D e IMRT ….......................... 14

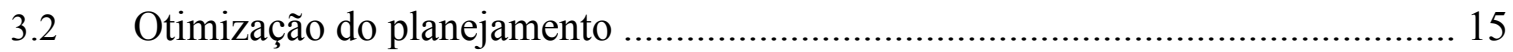

3.3 Métodos computacionais de cálculo da distribuição de dose .............................. 15

3.3.1 A interação dos fótons e o processo de deposição de energia ............................... 15

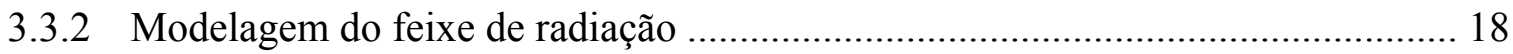

3.3.3 Cálculo de dose - algoritmo tipo Pencil Beam Convolution ................................ 19

3.4 Cálculo da dose absorvida por unidade monitora .............................................. 19

3.5 Comissionamento de um sistema de planejamento de IMRT .............................. 21

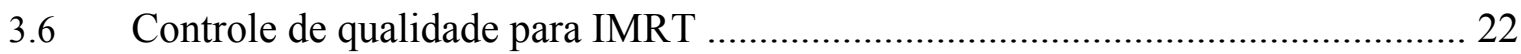

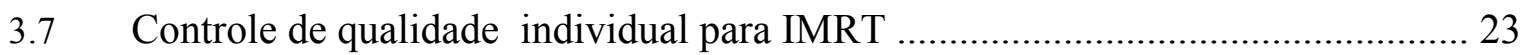

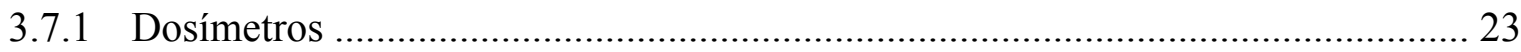

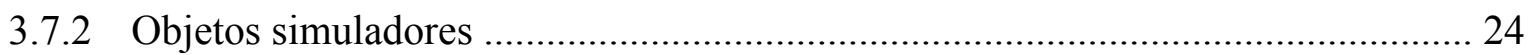

3.7.3 Comparação de distribuições de isodoses ............................................................. 24

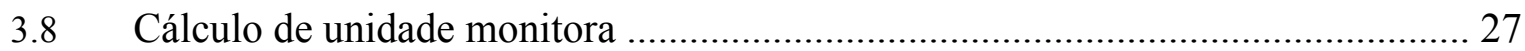

3.9 Método de integração de Clarkson ................................................................... 28

3.10 Métodos de cálculo de unidade monitora para IMRT …...................................... 30

3.11 Métodos independentes de cálculo de Unidade Monitora para IMRT .................. 31

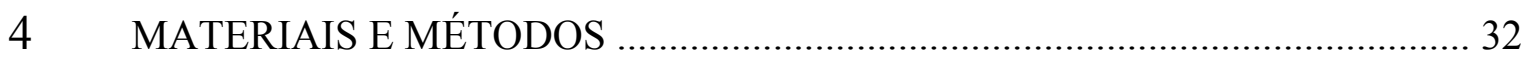

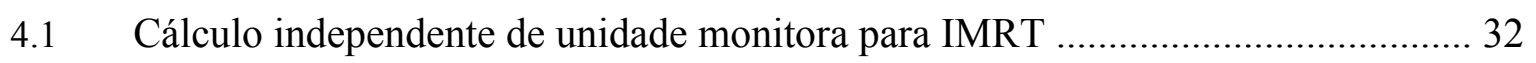

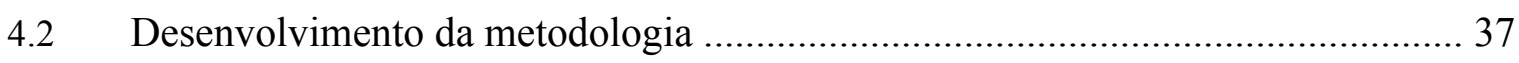

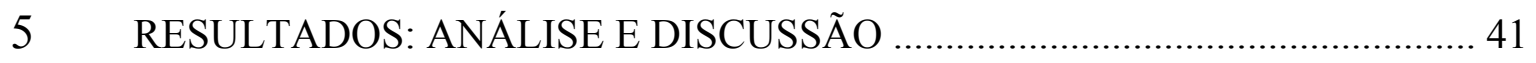

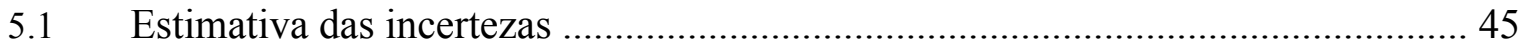

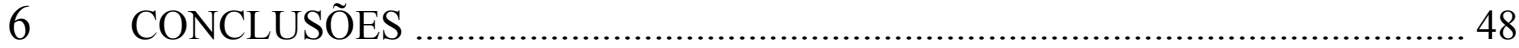

APENDICE A - Arquivo contendo o posicionamento das lâminas de MLC ................... 50

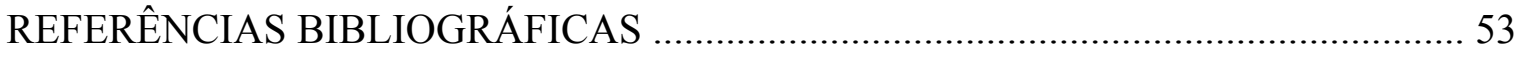




\section{LISTA DE FIGURAS}

Página

FIGURA 1 - Tela iterativa do processo de otimização do sistema de planejamento Eclipse ${ }^{\circledR}$

FIGURA 2 - Comparação entre duas técnicas de radioterapia: a) Conformacional 3D e b) IMRT

FIGURA 3 - Esquema padrão dos componentes do cabeçote de um acelerador linear, representando os colimadores secundários superiores e inferiores e os colimadores terciários de múltiplas lâminas. Reproduzida do trabalho de Boyer e col. (2001) no Task Group 50 16

FIGURA 4 - Influência do tamanho de campo na profundidade de dose em um objeto simulador heterogêneo no qual $\mathrm{A}$ é a região relacionada ao tecido adiposo, $\mathrm{M}$ ao músculo, B ao osso e L ao pulmão. Figura reproduzida de Ahnesjo e Aspradakis (1999) 17

FIGURA 5 - Imagem mostrando a superposição das isodoses calculadas através do sistema de planejamento e das isodoses medidas através de filme dosimétrico 25

FIGURA 6 - Conjunto dosimétrico bidimensional para comparação entre isodoses medidas e calculadas através do sistema de planejamento. O detalhe mostra a utilização da função gama e os critérios de aceitação pré-selecionados pelo usuário (\%diff é a porcentagem máxima aceita de diferença entre duas isodoses, $\mathrm{mm}$ a distância máxima em milímetros e $\%$ Pass é a porcentagem de pontos que respeitaram os critérios escolhidos)

FIGURA 7 - Exemplo de campo irregular com setores de Clarkson, extraída de Khan (2003)

FIGURA 8 - Distribuição da incidência dos 5 campos modulados utilizados em cada plano de tratamento: $135^{\circ}, 93^{\circ}, 0^{\circ}, 267^{\circ}, 225^{\circ}$

FIGURA 9 - Esquema ilustrativo do colimador de múltiplas lâminas (MLC) de 120 lâminas utilizado no estudo

FIGURA 10 - Campo modulado com resultado da subdivisão em 18 subcampos conformados com diferentes arranjos de MLC

FIGURA 11 - Fluência modulada do campo de tratamento como somatória de fluências planas de cada subcampo e da componente de transmissão através das lâminas

FIGURA 12 - Fluxograma do algoritmo de cálculo desenvolvido

FIGURA 13 - Histograma do desvio relativo de cada subcampo, num total de 20 subcampo calculados 


\section{LISTA DE TABELAS}

Página

TABELA 1 - Tabela de dados gerados a partir do sistema de planejamento para IMRT Caso 1

TABELA 2 - Planilha contendo dados individualizados para um campo e seus respectivos subcampos. No exemplo desta tabela, os dados são referentes ao campo 3 do caso $1 \mathrm{em}$ estudo 42

TABELA 3 - Detalhamento do cálculo das incertezas, baseado no TRS-398 46

TABELA 4 - Dose calculada independente com respectiva incerteza 46 


\section{LISTA DE ABREVIATURAS}

DMLC Dynamic Multileaf Collimator

DTA Distance to Agreement

DVH Dose Volume Histogram

EORTC European Organisation of Research and Treatment of Cancer

ESTRO European Society for Therapeutic Radiology and Oncology

ICRU International Commission on Radiation Units

IMAT Intensity Modulated Arc Therapy

IMRT Intensity Modulated Radiotherapy

MLC Multileaf Collimator

NTCP Normal Tissue Complication Probability

OAR Organ at Risk

PET Positron Emission Tomography

PSF Peak Scatter Factor

RTC-3D Radioterapia Conformada 3D

SF $\quad$ Scatter Factor

SMLC Segmented Multileaf Collimator

SMR Scatter Maximum Ratio

SPR Scatter Phantom Ratio

SPECT Single Photon Emission Computed Tomography

SSD Source Skin Distance

TLD Thermoluminescence Dosimetry

TMR Tissue Maximum Ratio

TPR Tissue Phantom Ratio

TPS Treatment Planning System

UM Unidade Monitora 


\section{INTRODUÇÃO}

O uso da radioterapia para a erradicação ou controle da doença causa danos tanto nos tecidos normais quanto no volume tumoral. A intensidade modulada do feixe de radiação atualmente é uma das técnicas mais avançadas de radioterapia conformada que promete aperfeiçoar os tratamentos aumentando a dose de radiação no volume tumoral e diminuindo a dose em tecidos sadios, elevando assim a probabilidade de controle do tumor e diminuindo, portanto a probabilidade de complicações no tecido normal (NTCP).

Diversas formas de radioterapia com intensidade modulada (IMRT) vêm sendo desenvolvidas nos últimos anos, desde um simples filtro compensador até métodos de varredura do feixe de radiação. Atualmente, a forma mais comumente utilizada para modulação do feixe de radiação é baseada na utilização de colimadores de múltiplas lâminas (Boyer e colaboradores, 2001b).

Técnicas de tratamento de IMRT têm sido clinicamente utilizadas desde os anos 60 , no entanto, as mais modernas só começaram a serem desenvolvidas no final dos anos 90 com a consolidação de imagens médicas tridimensionais, entre elas a tomografia computadorizada, a ressonância magnética, o PET e o SPECT, assim como o desenvolvimento de técnicas de controle de qualidade para verificação da dose e controle de tratamento de forma computadorizada.

A aplicação clínica do IMRT ainda apresenta algumas dificuldades não só devido à complexidade do equipamento utilizado para a administração da dose no paciente, como também da complexidade do processo de otimização da distribuição de dose feito por um software comercial e ao controle de qualidade relacionado com o cálculo da distribuição e entrega da dose, segundo Bayouth e colaboradores (2003).

Os sistemas de planejamento de tratamento computadorizados frequentemente utilizados nos serviços de radioterapia são responsáveis pelo cálculo das unidades monitoras para a liberação da dose prescrita. Estes sistemas de planejamento empregam métodos e grandezas diferentes daquelas usadas nos cálculos manuais das unidades monitores (UM). Com o atual desenvolvimento tecnológico crescente em radioterapia, 
sobretudo no que concerne o tratamento de pacientes com IMRT, é imprescindível o desenvolvimento de ferramentas adicionais de garantia da qualidade a fim de assegurar que o tratamento seja feito corretamente, conforme prescrição médica.

A dose calculada pelo sistema de planejamento requer um método independente de verificação para a liberação segura do tratamento em radioterapia e o cálculo de dose independente é o método mais apropriado para tal finalidade (Kuthcher e colaboradores (1994), Fraas e colaboradores (1998), Ezzell e colaboradores (2003)). Para a radioterapia de intensidade modulada (IMRT), onde o feixe de fótons pode ser altamente modulado e atenuado significativamente para alvos complexos, a verificação teórica da dose calculada pelo sistema de planejamento não é viável sem que haja um modelo detalhado das propriedades do feixe (Kung e colaboradores (2000), Jang e colaboradores (2006a)).

Por este motivo é desejável desenvolver um algoritmo confiável de cálculo de dose independente baseado em um modelo detalhado do feixe. Tal algoritmo deve considerar todos os efeitos potencialmente relevantes do colimador de múltiplas lâminas (MLC) a fim de prever seu impacto na liberação da dose para uma técnica de planejamento específica, objetivo de otimização, algoritmo de otimização e algoritmo de movimento das lâminas (Lorenz e colaboradores (2007)). 


\section{OBJETIVOS}

\section{Geral}

Desenvolver uma metodologia resultando num algoritmo para cálculo de dose independente para verificação dos tratamentos com Radioterapia de Intensidade Modulada (IMRT).

\section{Específico}

Validação da metodologia a partir de testes com dados de um sistema de planejamento comercial e de um acelerador linear Varian $21 \mathrm{iX}$ e a verificação manual do cálculo de dose. 


\section{REVISÃO DA LITERATURA}

$\mathrm{Na}$ radioterapia convencional alguns modificadores de feixes, tais como cunhas ou compensadores são utilizados para corrigir possíveis irregularidades de contorno ou até mesmo superfícies curvadas do paciente, garantindo uma melhor homogeneidade de dose no volume alvo a ser tratado.

O conceito de IMRT se desenvolveu com base em testes em algoritmos de otimização de planejamento de radioterapia que previam que campos de radiação ótimos eram tipicamente não uniformes (Brahme e colaboradores (1982); Brahme (1987 e 1988); Cormack (1987)). Mostrou-se que um grupo de feixes com modulação de intensidade poderia produzir homogeneidade de dose no volume tumoral similar à radioterapia convencional, mas com maior conformação, especialmente para volumes com formas côncavas ou complexas.

O cálculo da fluência de dose do feixe somente tornou-se prático através do desenvolvimento de computadores de alta capacidade e a utilização de algoritmos que realizam uma aproximação iterativa chamada de planejamento inverso.

O planejamento inverso inicia-se com a descrição das características da distribuição de dose desejada, como por exemplo: a dose mínima aceita no volume alvo; a dose máxima absorvida em um determinado órgão de risco (OAR); e as especificações que relacionam dose e volume para o tumor e os órgãos de risco envolvidos, junto com os fatores de ponderação de descrevem a importância relativa de cada estrutura. Estas características são incorporadas em uma função matemática, chamada de função objetivo. O procedimento de otimização, que é a busca iterativa para a solução que minimiza o custo e maximiza os benefícios, é guiado pela função objetivo.

Durante o planejamento do tratamento os valores descritos precisam ser ajustados pelo operador de forma a obter o melhor acordo entre objetivos diferentes, o que modifica consideravelmente a função objetivo. Por causa da natureza iterativa de encontrar a melhor solução e a necessidade de alterar os valores iniciais, utiliza-se o termo planejamento otimizado ao invés de planejamento inverso. Na Figura 1 é mostrado a tela 
de fatores de ponderação para o processo de otimização do sistema de planejamento do software Eclipse V.8.6 (Varian Medical System, Palo Alto, CA).

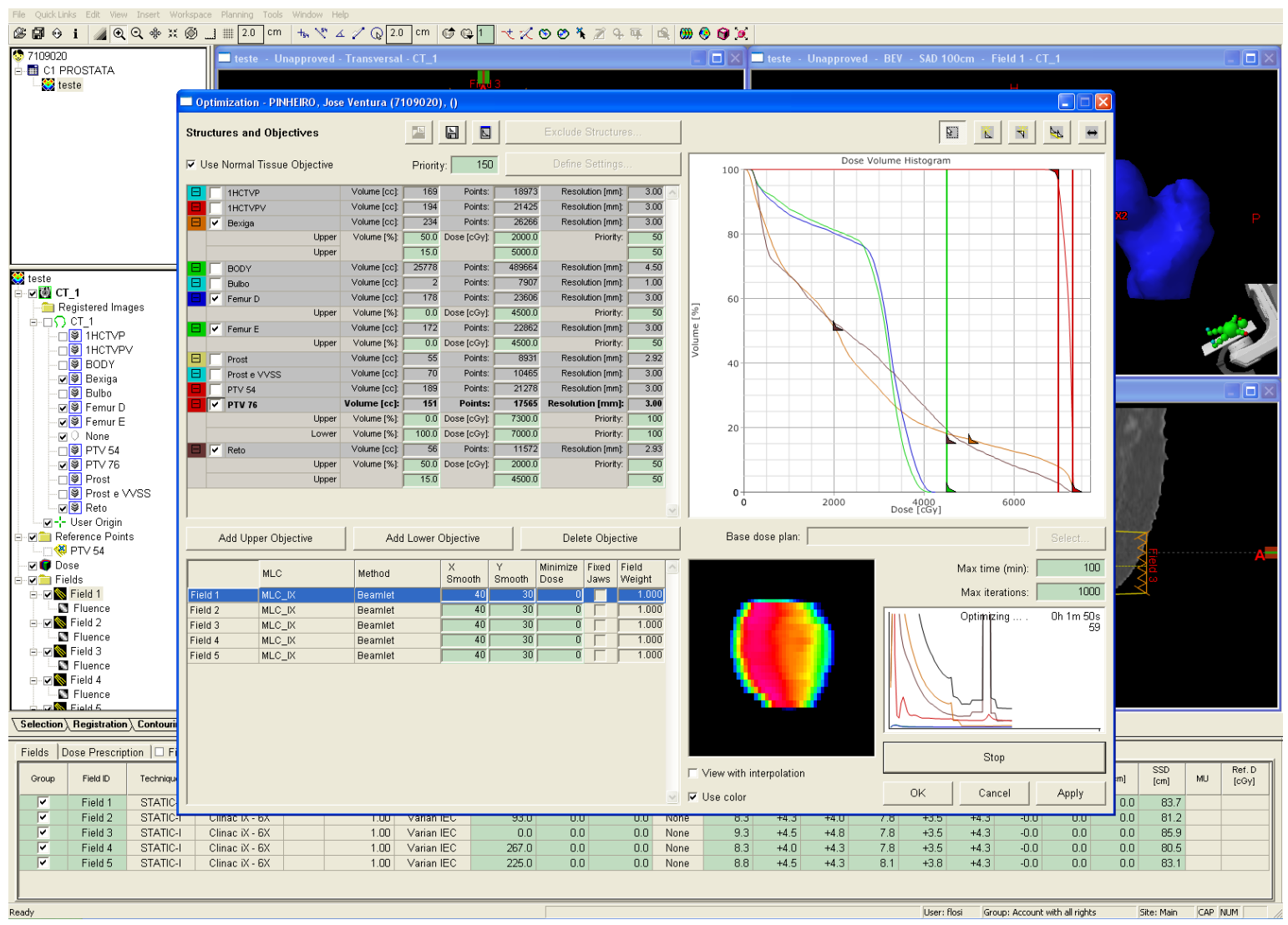

Figura 1 - Tela do processo de otimização do sistema de planejamento Eclipse ${ }^{\circledR}$.

Há diversas maneiras de obter feixes de radiação com intensidade modulada. $\mathrm{O}$ método mais usual é a utilização de colimadores de múltiplas lâminas (MLC). Os tratamentos de IMRT que utilizam MLC e angulações de tratamento de campos estáticos podem utilizar um dos métodos de entrega de dose descritos abaixo:

- Conformação segmentada das lâminas (SMLC), no qual o feixe é administrado com uma seqüência de pequenos segmentos ou subcampos, cada um com uma intensidade uniforme, ou seja, o feixe é ligado apenas quando as lâminas estão posicionados nos locais planejados pra cada subcampo e só há movimentação quando o feixe está desligado. Também conhecida como step and shoot (Bortfeld e colaboradores (1994); Siochi, (1999));

- Conformação dinâmica das lâminas (DMLC), no qual as lâminas se movimentam durante a irradiação do paciente. Também conhecido como 
sliding window (Convery e Rosenbloom (1992); Dirkx e colaboradores (1998); Spirou e Chui (1994));

Uma nova forma de tratamento recentemente proposta foi o arco dinâmico com intensidade modulada (IMAT). Nesta técnica, além da conformação dinâmica das lâminas, o gantry, a mesa de tratamento e os colimadores rotacionam em torno do paciente, podendo resultar numa distribuição de dose mais conformada (Yu (1995)).

\subsection{Comparação entre radioterapia conformacional 3D e IMRT}

$\mathrm{Na}$ radioterapia conformacional 3D (RTC-3D), alterações nas características primárias do feixe incluem a utilização de acessórios modificadores do feixe, tais como cunhas ou compensadores, ou fração de contribuição de dose absorvidas distintas para cada campo (Podgorsak e colaboradores (2005)).

A qualidade e o aperfeiçoamento das distribuições de dose absorvida e dos histogramas de dose por volume resultantes são altamente dependentes do operador. Este aperfeiçoamento é realizado através de modificações do feixe, tais como: o ângulo de incidência; o ângulo de rotação do colimador; a fração de contribuição de cada campo; e os limites do campo.

A radioterapia tridimensional transforma-se em IMRT quando cada campo de tratamento é subdividido em pequenos campos (subcampos) de intensidades não uniformes, ou através de múltiplos campos de intensidade uniforme e dimensões diferentes entregues numa mesma posição.

O planejamento de IMRT emprega técnicas de otimização automatizadas. A Figura 2 ilustra uma comparação entre radioterapia conformacional 3D e IMRT. 
RTC-3D

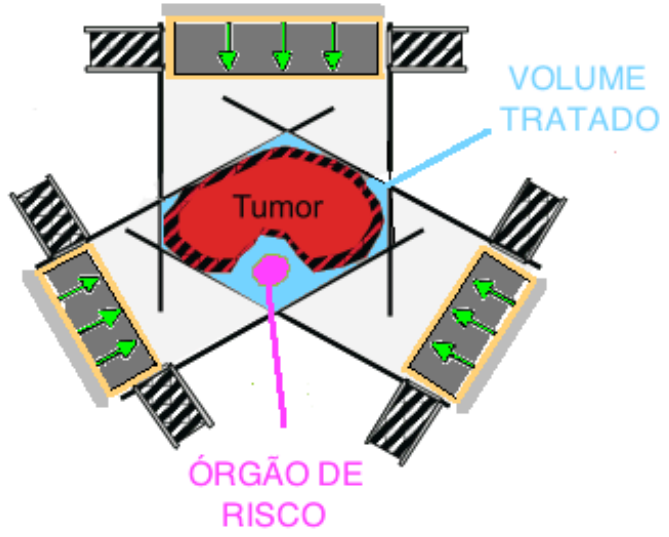

IMRT

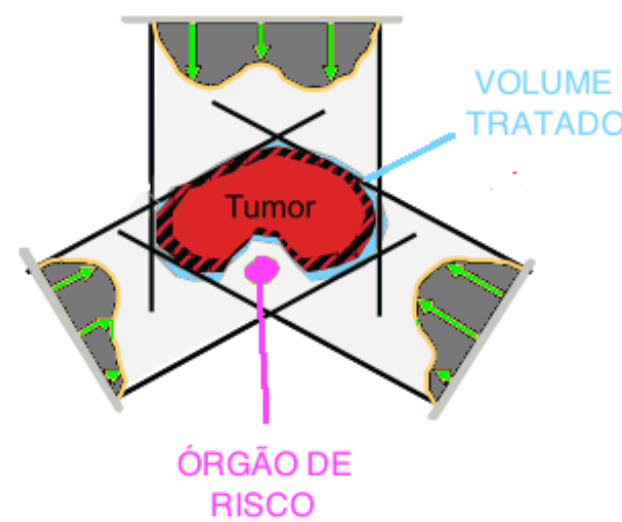

Figura 2 - Comparação entre duas técnicas de radioterapia: a) Conformacional 3D e b) IMRT.

\subsection{Otimização do planejamento}

No processo de otimização convencional escolhe-se primeiramente os parâmetros dos campos de tratamento, assim como a forma e a utilização de acessórios modificadores do feixe. Em seguida é realizado o cálculo computacional e avalia-se a distribuição de dose resultante em conjunto com o histograma de dose por volume (DVH). Os parâmetros dos campos são modificados iterativamente pelo operador, até que os objetivos sejam atingidos, como descrito por Boyer e colaboradores (2001b).

Já no processo de otimização de IMRT, define-se inicialmente os objetivos baseados em considerações do histograma de dose por volume. Através da utilização de funções matemáticas, chamada de funções objetivo, e da utilização de algoritmos computacionais iterativos, obtém-se uma solução ótima. Caso necessário, o operador altera os fatores de ponderação, que definem a função objetivo, conduzindo a um novo ciclo de otimização para atingir a melhor distribuição de dose.

\subsection{Métodos computacionais de cálculo da distribuição de dose}

\subsubsection{A interação dos fótons e o processo de deposição de energia}

A radioterapia com intensidade modulada do feixe (IMRT) exige mais atenção com relação aos diversos aspectos dos modelos computacionais utilizados para obtenção da distribuição de dose no paciente em relação à radioterapia convencional. 
O processo de produção e modelagem do feixe no aparelho de tratamento e a subsequente deposição de energia no paciente são muito complexos. O feixe que incide sobre o paciente é composto pelos fótons gerados no alvo de raio X, que alcança o paciente sem interação com as componentes da unidade de tratamento, chamada de radiação direta, pelos fótons que interagem com o cabeçote da unidade de tratamento, chamada de radiação indireta e pela contaminação eletrônica oriunda da interação dos fótons com os componentes do cabeçote do equipamento. Na Figura 3 estão representados os componentes de um acelerador linear padrão com a inclusão dos colimadores de múltiplas lâminas já considerados assim como colimadores terciários.

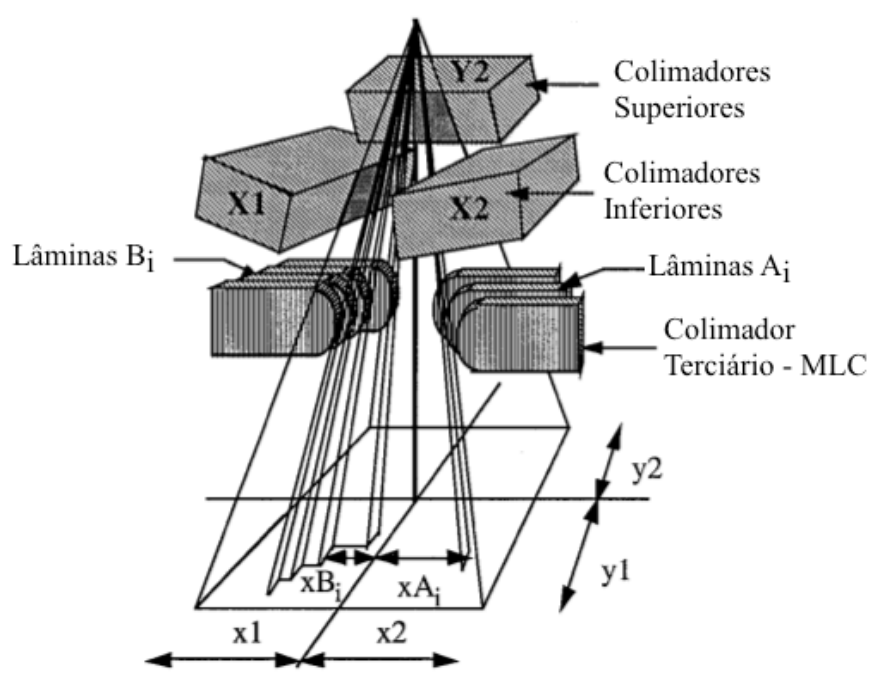

Figura 3 - Esquema padrão dos componentes do cabeçote de um acelerador linear, representando os colimadores secundários superiores e inferiores e os colimadores terciários de múltiplas lâminas. Reproduzida do trabalho de Boyer e col. (2001) no Task Group 50.

Segundo Ahnesjö (1994) e Liu e colaboradores (1997), os fótons espalhados são responsáveis por cerca de $2 \%$ a $15 \%$ da dose total absorvida no paciente, dependendo da energia do feixe e do projeto do cabeçote. Estes fótons atuam como fontes alargadas, sendo responsáveis pelo alargamento da região de penumbra do campo. Colimadores primários do feixe convencionalmente são paralelos à divergência do campo a partir da fonte, a fim de reduzir a produção dos fótons indiretos.

Alguns colimadores de múltiplas lâminas (MLC), que são colimadores terciários, responsáveis pelo formato e modulação do feixe de radiação, possuem as bordas arredondadas. Este tipo de formato impede que haja uma descontinuidade entre a projeção da luz e o campo radiação. Comparado com as lâminas com borda divergente, as lâminas 
de borda arredondada aumentam a porcentagem de transmissão dos fótons, causam um limite mais borrado do campo (região de penumbra) e produzem maior quantidade de fótons indiretos e contaminação eletrônica (Boyer e colaboradores (2001a), Task Group $50)$.

O algoritmo de cálculo computacional deve ser capaz de levar em consideração todas as características do espectro do feixe produzido, sendo bastante importante no caso de IMRT, que a modelagem da penumbra do feixe, da transmissão de radiação através e entre as lâminas dos colimadores de múltiplas lâminas, e da contaminação do feixe com fótons indiretos e elétrons, seja a mais precisa possível, como recomendado pelo ICRU Report $n^{\circ} 83$.

Quando o tamanho de campo é pequeno, como nos casos de IMRT, para pequenas variações no tamanho ocorrem grandes alterações na dose absorvida. A influência do tamanho de campo na porcentagem de dose absorvida em função da profundidade é mais significativa quanto maior a energia do feixe de fótons incidente, indicando que a radioterapia com modulação de intensidade do feixe exige a habilidade de determinar exatamente a dose absorvida para campos pequenos, especialmente em regiões heterogêneas.

A Figura 4 mostra os dados computacionais gerados por um algoritmo de convolução / superposição da dose absorvida por fluência de energia na direção do eixo central de um feixe de $4 \mathrm{MV}$ em função da profundidade e diâmetro do feixe.

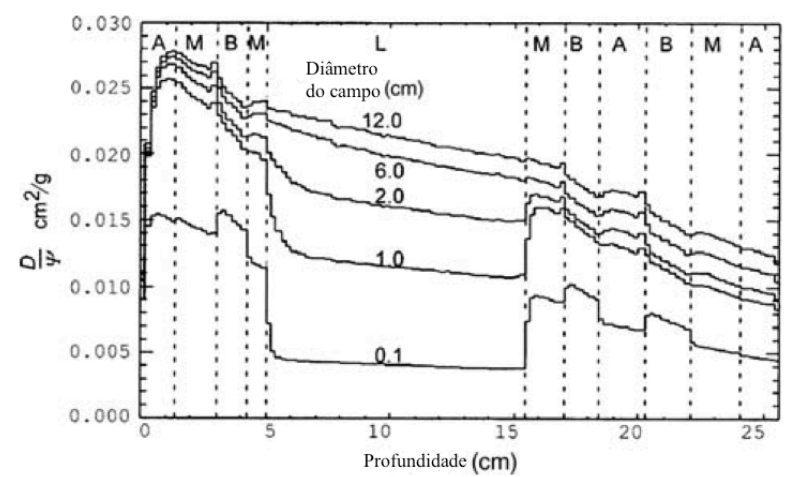

Figura 4 - Influência do tamanho de campo na profundidade de dose em um objeto simulador heterogêneo no qual A é a região relacionada ao tecido adiposo, $\mathrm{M}$ ao músculo, $\mathrm{B}$ ao osso e $\mathrm{L}$ ao pulmão. Figura reproduzida de Ahnesjo e Aspradakis (1999). 


\subsubsection{Modelagem do feixe de radiação}

Segundo a publicação do ICRU Report $n^{\circ} 83$ existem duas classes principais de algoritmos computacionais de cálculo da distribuição de dose: baseados em correções e baseados em modelos.

Em algoritmos baseados em correções, a dose absorvida é primeiramente medida em um objeto simulador de água com um feixe retangular incidindo perpendicularmente a superfície do objeto simulador e depois a distribuição de dose é parametrizada em função do tamanho de campo, profundidade, e da distância entre a fonte e a superfície do objeto simulador. Esta parametrização é então corrigida para perturbações específicas, tais como: presença de blocos de colimação do feixe; cunhas ou compensadores de tecido; para superfícies irregulares e heterogeneidade de tecido.

Nas aproximações baseadas em modelo, as medidas das distribuições de dose para uma variedade de situações são utilizadas para desenvolvimento de parâmetros para um modelo que deve descrever a atenuação dos fótons incidentes e a produção da radiação secundária.

Um modelamento do feixe usual deve ser simples para entender, comissionar e implementar.

Para o modelamento de propriedades mais complexas, assim como as variações da penumbra, é necessário um modelo mais elaborado. Foram desenvolvidos modelos que consideram fontes múltiplas e também o espalhamento do feixe através de todos os componentes do cabeçote (Liu e colaboradores (1997)). Tipicamente a fonte primária é tratada com uma distribuição gaussiana originada de duas fontes.

Existem também os modelos baseados em código de Monte Carlo que permitem simulações detalhadas do cabeçote de tratamento (Rogers e colaboradores (1995)). Os resultados das simulações de Monte Carlo são tipicamente de um "espaço de fase", isto é, uma série de dados que fornecem uma lista da energia e do sentido dos milhões de partículas emitidas. 


\subsubsection{Cálculo de dose - algoritmo tipo Pencil Beam Convolution}

O cálculo da dose com o algoritmo Pencil Beam Convolution (PBC) é divido em duas partes principais: modelamento da reconstrução do feixe e modelamento do paciente.

No modelamento da reconstrução do feixe, a dose é primeiramente obtida em um meio homogêneo e equivalente à água. Todos os campos adicionados, assim como, blocos de colimação, filtros, colimados de múltiplas lâminas e compensadores que modificam o formato ou modulam a intensidade do feixe de radiação são levados em consideração. A dose é calculada com o algoritmo de PBC, que é um algoritmo de convolução baseado em pencil beam kernels (Storchi e colaboradores, 1999).

A utilização do PBC para o cálculo da dose no volume inteiro de interesse é um processo muito demorado, dessa forma, a convolução é usada para calcular a dose em cinco planos perpendiculares à incidência do feixe, e a dose para todos os outros pontos do volume é obtida através da interpolação destes planos. Na primeira etapa do processo é utilizada uma geometria padrão em um objeto simulador equivalente a àgua.

Uma vez que a dose foi reconstruída, a etapa seguinte é a transformação para uma geometria que representa a distância verdadeira do eixo central do foco do campo à pele do paciente. Esta transformação é feita através de métodos padrões: fator de Mayneord e correção de TAR.

Após as etapas da reconstrução do feixe, realiza-se o modelamento da curvatura real da pele e das heterogeneidades do paciente. Segundo Sievinen e colaboradores (2005) o algoritmo PBC calcula a distribuição de dose no paciente com as seguintes incertezas:

- $\quad$ Entre 2 e $3 \%$ para feixe de fótons padrão;

- Para modelos de reconstrução do feixe de fótons, aproximadamente $1 \%$ para campos retangulares e $2 \%$ para campos irregulares;

- Entre 1 e 2\% para campos com correção oblíqua.

\subsection{Cálculo da dose absorvida por unidade monitora}

As unidades monitoras se referem à leitura da corrente integrada de uma câmara de ionização de placas paralelas do acelerador linear, sendo que esta leitura é 
proporcional à intensidade média do feixe. As câmaras de ionização utilizadas como monitoras em feixes de fótons de alta energia normalmente são seladas de modo que não haja variações na leitura devido aos efeitos da pressão e da temperatura.

A dose absorvida por unidade monitora é calibrada em função da dose absorvida medida em um objeto simulador sob condições de referência especificadas em protocolos internacionais, sendo mais comumente utilizado o protocolo da Agência Internacional de Energia Atômica (Andreo e colaboradores (2000)).

$\mathrm{Na}$ radioterapia convencional, parte do processo de planejamento é determinar a quantidade de unidades monitoras que será entregue por cada campo. Em IMRT, o significado difere ligeiramente dependendo do tipo de administração do feixe.

Para o método de entrega de dose por conformação segmentada das lâminas (SMLC), o cláculo das unidades monitoras para cada subcampo deve considerar o fator output, assim como as contribuições das fontes indiretas de radiação e de radiação espalhada pelo sistema de colimadores. E, para o método de conformação dinâmica das lâminas (DMLC), o cálculo das unidades monitoras se refere a quantidade de ionização acumulada durante o tempo total do tratamento dinâmico, exigindo uma sincronização cuidadosa das lâminas, de modo que o padrão das lâminas seja finalizado exatamente quando as unidades monitoras totais forem entregues.

Em aceleradores lineares convencionais, a dose absorvida por unidade monitora, $\frac{D}{U M}$, é descrita pelas equação 1 , segundo o ICRU Report $\mathrm{n}^{\circ} 83$ :

$$
\frac{D(A, r)}{U M}=\frac{\Psi_{0}}{U M} \cdot[1+b(a)]^{-1} \cdot \frac{D(A, r)}{\Psi_{0}}
$$

em que $\Psi_{0}$ é a fluência de energia $D(A, r)$ é a dose absorvida para um campo de tamanho $A$ e profundidade $r$.

$$
\frac{\Psi_{0}}{U M}=\frac{\left[D\left(A_{\text {cal }}, r_{\text {cal }}\right) / U M\right]_{\text {medido }}}{\left[D\left(A_{\text {cal }}, r_{\text {cal }}\right) / \Psi_{0}\right]_{\text {calculado }}} \cdot\left[1+b\left(A_{\text {cal }}\right)\right]
$$

em que o índice cal denota a variável referente à geometria de calibração do feixe (normalmente em um objeto simulador de água com o isocentro localizado à $10 \mathrm{~cm}$ de profundidade e um campo quadrado de tamanho 10 x $10 \mathrm{~cm}$ ). 
O fator $\left[1+b\left(A_{c a l}\right)\right]$ corrige variações relacionadas ao retro espalhamento dos fótons na câmara monitora. Como mostrada pela Equação 2, a fluência da energia por unidade monitora é obtida através da razão da medida da dose absorvida por unidade monitora sob condições de calibração dividida pela dose absorvida por fluência de energia calculada pelo sistema de planejamento sob as mesmas condições da calibração.

\subsection{Comissionamento de um sistema de planejamento de IMRT}

O objetivo do comissionamento do sistema de planejamento é assegurar que o cálculo da distribuição de dose represente de forma mais realisticamente possível o que acontece no paciente propriamente dito.

Segundo Khan (2003), os passos envolvidos no comissionamento de um sistema de IMRT dependerão principalmente do tipo de sistema de planejamento inverso utilizado. Alguns sistemas de planejamento inverso são módulos simples dentro do sistema de planejamento tridimensional padrão e utilizam o algoritmo de cálculo regular para avaliar a dose a partir dos mapas de fluência otimizados. Para comissionar este tipo de sistema é necessário primeiramente comissionar o sistema de planejamento padrão, e depois realizar medidas adicionais de caracterização das propriedades básicas dos colimadores de múltiplas lâminas (MLC) (transmissão do feixe de radiação através das lâminas e na junção entre cada duas lâminas, velocidade máxima de movimentação e outros parâmetros específicos). Outros algoritmos são sistemas individuais que precisam dos dados completos, medidos ou modelados, separados do sistema de planejamento tridimensional.

A maioria dos sistemas de planejamento de IMRT usam algoritmos de otimização que envolvem cálculos iterativos. A técnica de IMRT pode produzir regiões de alto gradiente nas distribuições de dose, devido à proximidade do volume alvo e dos órgãos de risco, portanto os testes durante o comissionamento são críticos para assegurar resultados precisos dos sistemas de planejamento.

Cada método de entrega de dose deve ser comissionado separadamente, além do cálculo e determinação das características das lâminas, tais como a transmissão intralâminas, entre lâminas e a descrição do formato das bordas para definir exatamente a região de penumbra e verificar a acurácia e reprodutibilidade do posicionamento e 
movimentação das lâminas (Graves e colaboradores (2001); Hardcastle e colaboradores (2007)).

Segundo ICRU Report $\mathrm{n}^{\circ} 83$ (2010), a técnica de IMRT resulta em um incremento de 2 à 10 vezes mais unidades monitoras que a técnica convencional.

\subsection{Controle de qualidade para IMRT}

Atualmente existem evidências crescentes indicando que o alto grau de exatidão na entrega de dose está associado ao sucesso nos tratamentos de radioterapia. Estudos da European Organisation of Research and Treatment of Cancer (EORTC) mostram que a exatidão na dosimetria clínica pode ser mantida se os seguintes níveis de aceitação forem adotados (Johansson e colaboradores (1986)):

- $\quad \pm 3 \%$ para calibração das unidades de tratamento, e

- $\quad \pm 5 \%$ para entrega da dose prescrita (na qual estão envolvidos características das colimações do feixe, profundidade de tratamento, bandejas, filtros, sistemas de planejamentos, entre outros).

Para um tratamento efetivo de radioterapia convencional, o objetivo é que incerteza total na dose absoluta entregue ao paciente não seja maior que 5\%, segundo publicações e estudos de referência feitos no ICRU $n^{\circ}$. 24 (1976), por Kutcher e colaboradores no Task Group 40 (1994) e, posteriormente, incluindo técnicas mais atuais como o IMRT, no ICRU n ${ }^{\circ} .83$ (2010).

Para IMRT, Van Dyk e colaboradores (1993) sugeriram que a exatidão dependesse do gradiente de dose absorvida. Em regiões de baixo gradiente, a diferença relativa entre a dose calculada pelo sistema de planejamento e a dose medida é mais importante. Entretanto em regiões de gradiente alto a distância entre as isodoses de mesmo valor medidas e calculadas através do sistema de planejamento torna-se mais importante, uma vez que, nas regiões de gradiente alto, a variação no valor das isodoses se torna muito grande em relação a pequenas variações na distância entre elas. A sugestão para regiões de baixo gradiente foi um nível de exatidão dentro de $\pm 4 \%$, e para regiões de alto gradiente, foi um nível de acurácia dentro $\pm 3 \%$ com inclinações menores de $\pm 20 \% / \mathrm{cm}$ e distância entre as isodoses de $4 \mathrm{~mm}$. 
O cálculo computacional da dose absorvida administrada pelos campos pequenos de IMRT depende dos dados medidos e/ou modelados, tais como: a medida da região de penumbra; fator output; fator offset, que corrige a discrepância das lâminas entre o campo luminoso e o campo radioativo; fator transmissão inter e intra-lâminas; e exatidão dosímento e posicionamento das lâminas.

\subsection{Controle de qualidade individual para IMRT}

Para administração de dose na técnica de IMRT, o número de unidades monitoras (UM) exigido é uma função de uma sequência complexa, tanto para sequências dinâmicas quanto para sequências estáticas das lâminas, baseado na geometria do paciente e do volume alvo, sendo consequentemente imprevisível, aumentando a chance de ocorrer um erro sem um mecanismo apropriado de validação quantitativa independente, Low e colaboradores (2011).

Medidas diretas da dose, através da utilização de objetos simuladores, ou de softwares comerciais de cálculo independente da dose, são necessários para validar o plano de tratamento de cada paciente específico.

\subsubsection{Dosímetros}

Para a medida dosimétrica da dose absorvida e da sua distribuição, os dosímetros mais utilizados comercialmente são basicamente de dois tipos: pontuais (câmaras de ionização, diodos e TLD) e bidimensionais (filmes dosimétricos, conjunto de detectores, e radiografias digitais), em conjunto com diversos tipos de objetos simuladores, conforme o objetivo da medida, como revisado no trabalho de Low e colaboradores (2011).

Câmaras de ionização são utilizadas para medidas de fótons de megavoltagem devido a sua estabilidade excelente, resposta linear, dependência direcional pequena, independência da qualidade do feixe e rastreabilidade da calibração com relação a um padrão primário.

Diodos (semicondutores tipo p) tipicamente possuem volumes ativos muito pequenos e alta sensibilidade para radiação, ideal para medidas de campos pequenos, porém possuem dependência direcional. 
Um outro tipo de dosímetro para campos pequenos utilizados em IMRT é o dosímetro termoluminescente (TLD). O TLD é um dosímetro de integração, usualmente em forma de pastilhas cúbicas ou cilíndricas, com composição atômica aproximadamente tecido equivalente. Tipicamente apresenta resposta não linear com a dose, e deve ser avaliado antes da utilização.

A dosimetria pontual determina a dose absoluta em pontos individuais, mas a validação e o aceite da qualidade do plano de IMRT requer uma dimensão adicional da distribuição de dose, ou seja, sistemas dosimétricos 2D. As opções comerciais atuais são os filmes dosimétricos, filmes radiocromicos, radiografias digitais, conjuntos bidimensionais de detectores de diodo e conjuntos bidimensionais de câmaras de ionização.

\subsubsection{Objetos simuladores}

O tipo de objeto simulador utilizado no processo de verificação de IMRT, varia conforme o objetivo da medida. Os objetos simuladores são tipicamente construídos usando água ou material equivalente a água, em geometria cúbicas, cilíndricas ou antropomórficas. Alguns objetos simuladores podem conter materiais com densidades diferentes para simulação e medidas de meios heterogêneos.

\subsubsection{Comparação de distribuições de isodoses}

Os sistemas de planejamento calculam a distribuição de dose tridimensional de onde é exportado uma distribuição planar para comparação com a distribuição medida. Ambas são representadas em um sistema de coordenadas coincidente e comparadas em relação a distância entre duas isodoses coincidentes e em relação ao valor medido. A Figura 5 mostra a comparação entre a distribuição calculada pelo sistema de planejamento e a distribuição obtida através da irradiação de um filme dosimétrico. Existem diversos trabalhos neste assunto, entre eles Low e colaboradores (2003), Bakai e colaboradores (2003) e Stock e colaboradores (2005). 


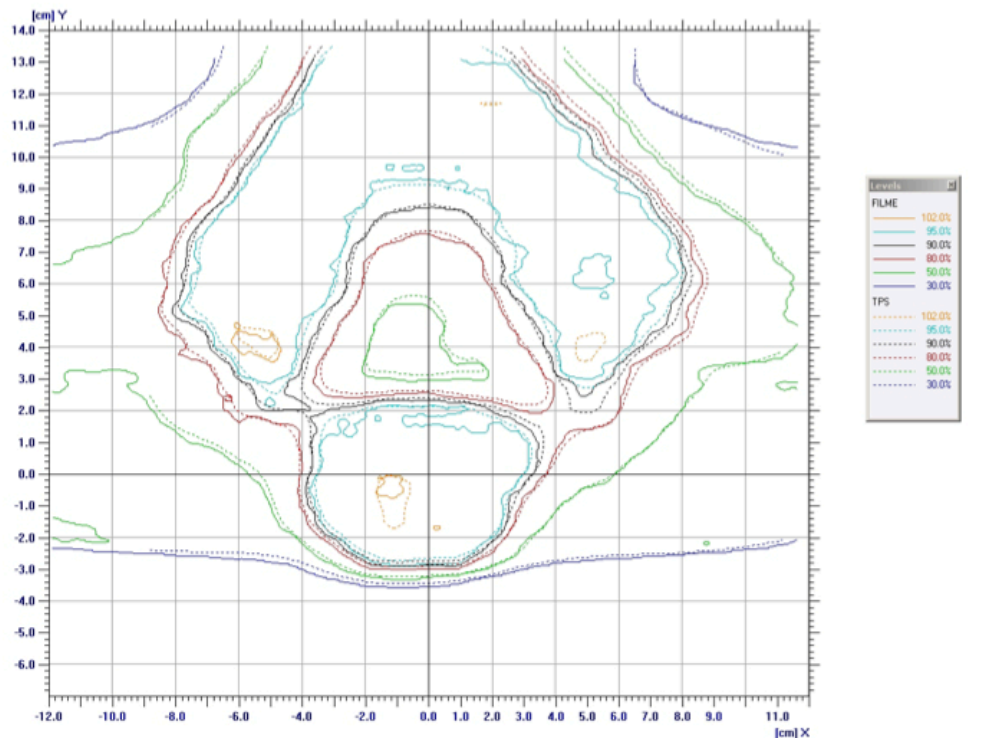

Figura 5 - Imagem mostrando a superposição das isodoses calculadas pelo sistema de planejamento e das isodoses medidas através de filme dosimétrico.

O método usual de comparação de isodoses num determinado plano exige que o usuário interprete as diferenças, detectando visualmente regiões onde as isodoses diferem. Este método é muito intuitivo, tendo como limitação que as grandes diferenças entre as isodoses podem ser causadas por discrepâncias espaciais, relacionadas ao posicionamento, relativamente pequenas nas regiões de alto gradiente de dose. Dado sua natureza quantitativa e intuitiva, este método deve ser empregado associado a utilização de outras ferramentas, tais como a análise através da função DTA (distance-to-agreement) e da função gama. Estas ferramentas de análise de distribuição de dose devem ser usadas para avaliar as medidas comparadas aos cálculos 3D.

A ferramenta de análise DTA foi desenvolvida para fornecer ao usuário uma medida da discrepância da distância entre duas distribuições de dose, Van Dyk e colaboradores (1993). A aplicação usual da função DTA é calcular a distância para cada ponto de referência, fazendo a varredura da distribuição para o ponto mais próximo que tem o mesmo valor de dose que o ponto de referência. Em regiões de gradiente de dose alto, isto pode ser interpretado como a distância entre as duas distribuições da dose. Esta interpretação é baseada na suposição que a distância é causada inicialmente por um deslocamento espacial entre as duas distribuições. Para as distribuições que diferem por dado deslocamento, a distribuição de DTA fornece uma medida exata deste valor, entretanto, a discrepância não pode ser relacionada a um erro do algoritmo ou da entrega da dose, mas simplesmente devido ao erro experimental. 
Quando a sobreposição das isodoses indica uma falha na posição, não há nenhuma indicação do valor da falha. Ter uma medida quantitativa do grau de acordo permite que o usuário desenvolva uma resposta apropriada ao grau de desacordo.

Low e colaboradores (1998 e 2003) desenvolveram uma ferramenta que combinasse critérios da dose e da distância em um único teste quantitativo, a função gama.

$\mathrm{Na}$ análise gama, as doses e as coordenadas espaciais são re-normalizadas por critérios pré-selecionados pelo usuário, e como são independentes, podem ser analisadas simultaneamente. Para cada ponto da distribuição de referência (calculado pelo TPS) a distância e a dose de cada ponto na distribuição a ser comparada (distribuição medida) é encontrada. A aproximação (distância) da distribuição de referência é chamada de valor gama. O valor gama é unitário quando a aproximação da distribuição de referência está dentro de uma esfera unitária, que indica a região dentro da qual o teste de comparação é satisfeito. Consequentemente, se a distribuição de referência está dentro deste limite o teste passa, caso contrário ele falha.

A Figura 6 mostra um conjunto dosimétrico bidimensional de câmaras de ionização e a comparação entre isodoses medidas e calculadas através do sistema de planejamento.

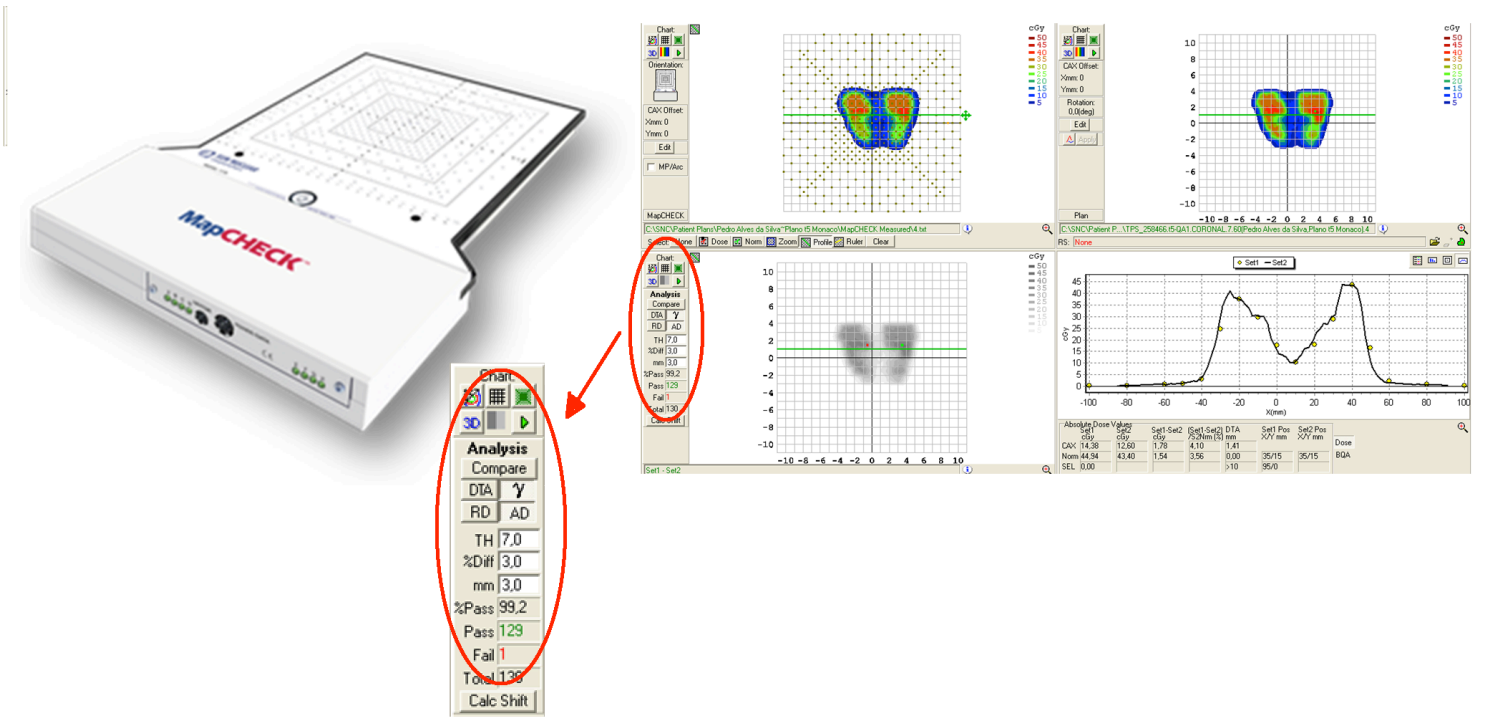

Figura 6 - Conjunto dosimétrico bidimensional para comparação entre isodoses medidas e calculadas através do sistema de planejamento. $O$ detalhe mostra a utilização da função gama e os critérios de aceitação pré-selecionados pelo usuário (\%diff é a porcentagem máxima aceita de diferença entre duas isodoses, $\mathrm{mm}$ a distância máxima em milímetros e \%Pass é a porcentagem de pontos respeitaram os critérios escolhidos). 
Nenhuma ferramenta de comparação fornece toda informação necessária para avaliação quantitativa da distribuições de dose. Cada ferramenta tem limitações que precisam de ser compreendidas ao conduzir as avaliações.

\subsection{Cálculo da Unidade Monitora}

Em 1997 a ESTRO (Booklet n³) publicou um documento descrevendo todas as medidas e cálculos necessários para a determinação da dose absorvida por unidade monitora (aceleradores lineares) ao longo do raio central. O cálculo de unidade monitora para métodos convencionais é definido pela equação 3:

$$
U M=\frac{\text { Dose }}{T M R(C E C) \times F_{t} \times \frac{\% I S O}{100}} \cdot \frac{\text { Peso }}{\sum \text { Peso }}
$$

em que $T M R(C E C)$ é a relação tecido máximo referente ao campo equivalente colimado, $F_{t}$ são todos os fatores modificadores do feixe, como por exemplo, fator bandeja, fator filtro, fator rendimento, entre outros, e \%ISO é o valor da isodose de prescrição do plano de tratamento. O termo $\frac{P e s o}{\sum P e s o}$ é o fator de ponderação que relaciona a porcentagem de contribuição de cada campo utilizado.

A relação tecido máximo é definida como a razão entre a dose, $D_{Q}$, ou taxa de dose, $\dot{D}_{Q}$, em um objeto simulador num ponto arbitrário $Q$ no raio central e a dose ou taxa de dose em um objeto simulador na profundidade de dose máxima, $z_{\text {máx }}$ definida a seguir:

$$
\operatorname{TMR}\left(z, A_{Q}, h v\right)=\frac{D_{Q}}{D_{Q_{\text {máx }}}}=\frac{\dot{D}_{Q}}{\dot{D}_{Q_{\text {máx }}}}
$$

A $\operatorname{TMR}\left(z, A_{Q}, h v\right)$ para um campo de tamanho $A_{Q}$ pode ser separada em uma componente primária e uma componente de espalhamento. A componente primária $\operatorname{TMR}(\mathrm{z}, 0, \mathrm{hv})$, supõe um campo hipotético de tamanho nulo em que a dose na profundidade Q é inteiramente devida aos fótons primários, uma vez que o volume para espalhamento da radiação é zero. E a componente de espalhamento, referida como relação espalhamento máximo, $\operatorname{SMR}\left(z, A_{Q}, h v\right)$, é definida por Podgorsak e col. (2005):

$$
\operatorname{SMR}\left(z, A_{Q}, h v\right)=T M R\left(z, A_{Q}, h v\right) \cdot \frac{S F\left(A_{Q}, h v\right)}{S F(0, h v)}-\operatorname{TMR}(z, 0, h v)
$$


em que $S F\left(A_{Q}, h v\right)$ e $S F(0, h v)$ são fatores de espalhamento para fótons de energia $h v$ e campos de tamanho $A_{Q}$ e zero, respectivamente. Estas componentes são definidas em função do fator espalhamento pico, $P S F$, que relaciona a dose medida na profundidade de máxima dose, $D_{p}$, e a dose medida no ar (mantendo as condições de equilíbrio eletrônico), $D_{p}^{\prime}$, mantendo o mesmo posicionamento. As equações são mostradas a seguir:

$$
\begin{aligned}
& \operatorname{SF}(A, h v)=\frac{\operatorname{PSF}(A, h v)}{\operatorname{PSF}(10, h v)} \\
& \operatorname{PSF}(A, h v)=\frac{D_{p}\left(z_{\text {máx }}, A, f, h v\right)}{D_{p}^{\prime}(A, h v)}
\end{aligned}
$$

O termo $P S F(10, h v)$ na Equação 6 representa o $P S F$ normalizado a 1 para um campo $10 \times 10 \mathrm{~cm}^{2}$.

A componente de espalhamento, relação espalhamento máximo, é utilizada no cálculo do campo equivalente colimado pelo método de integração de Clarkson.

\subsection{Método de integração de Clarkson}

A técnica de integração seccional foi criada por Clarkson (1941) e desenvolvida por Johns e Cunnigham nos anos 60 para a determinação da componente de espalhamento da dose em um ponto arbitrário de interesse no paciente, dentro ou fora do campo de radiação. Esta técnica se baseia no princípio de que a componente de espalhamento da dose no ponto, que depende do tamanho e da forma de campo, pode ser calculada separadamente da componente primária, que é independente do tamanho e da forma do campo.

Esta técnica transforma o campo irregular em setores de campos circulares originados no ponto de interesse $Q$ em um objeto simulador ou paciente.

Supõe-se que um setor de campo radial contribui $1 / N$ do valor do campo circular total para o valor de uma dada função $F$ para o campo irregular no ponto $Q$, na qual $N$ é o número total de setores num círculo (Podgorsak e colaboradores (2005)).

O valor da função $F$ para um campo irregular geralmente depende da profundidade $z$ do ponto $Q$, formato do campo irregular, SSD $=f$ e energia do feixe $h v$ e determinada pela seguinte relação: 


$$
F(z, \text { campo irregular, } f, h v)=\frac{1}{N} \sum_{i=1}^{N} F\left(z, r_{i}, f, h v\right)
$$

em que $r_{i}$ é o raio (do ponto $Q$ ao limite do campo) centrado em $i$, e $\mathrm{F}\left(\mathrm{z}, \mathrm{r}_{\mathrm{i}}, \mathrm{f}, \mathrm{h} v\right)$ é a função dosimétrica na profundidade $z, \operatorname{SSD}=f$ e energia do feixe $h v$ e raio do campo circular $r_{i}$.

Um exemplo de subcampo calculado é mostrado na Figura 7, com 2 setores destacados em negrito: um é um setor simples com raio $r_{1}$ e o outro é um setor composto com 3 raios: $r_{a}, r_{b}$ e $r_{c}$.

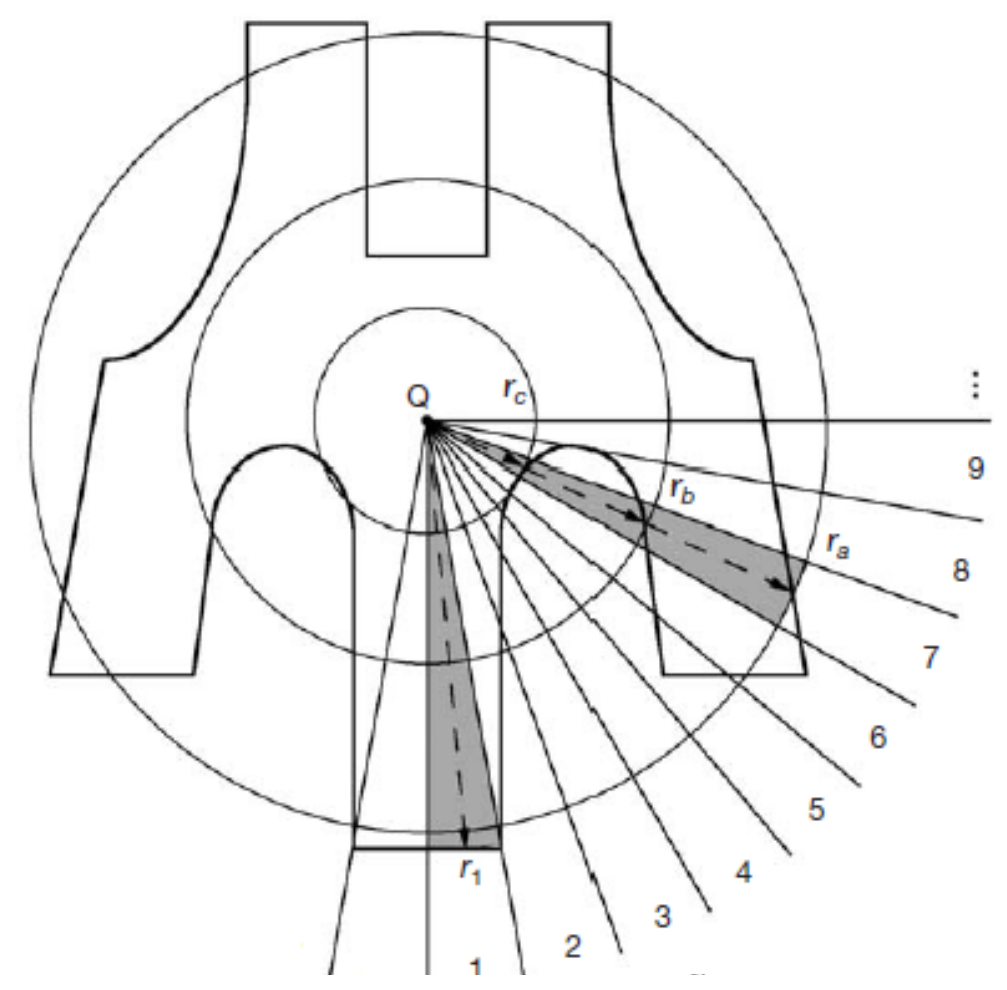

Figura 7 - Exemplo de campo irregular com setores de Clarkson, extraída de Khan (2003).

A contribuição do setor simples à somatória na Equação 8 é dado pelo seguinte termo:

$$
\left(\frac{1}{N}\right) F\left(z, r_{i}, f, h v\right)
$$

A contribuição na somatória dada na Equação 8 do setor composto de 3 componentes é dada pela equação 10 , na qual os termos positivos são contribuições da 
porções do campo de radiação e o termo negativo considera a perda da porção do campo de radiação no segmento, isto é:

$$
\left(\frac{1}{N}\right)\left[F\left(z, r_{a}, f, h v\right)-F\left(z, r_{b}, f, h v\right)+F\left(z, r_{c}, f, h v\right)\right]
$$

O valor da função de dose para um dado campo irregular é determinado através do método de Clarkson, e o campo equivalente quadrado está relacionado à esta função de dose, em que estes valores estão tabelados.

\subsection{Métodos de cálculo de Unidade Monitora para IMRT}

Quando o formato do campo de radiação é dado pela abertura do colimador, os fatores do espalhamento para os campos retangulares estão estimados calculando os campos quadrados equivalentes usando uma relação entre sua área e perímetro (Day e Aird (1983)). O fator espalhamento pelo colimador para um campo retangular é assumido como sendo o mesmo que do campo quadrado equivalente, que pode ser interpolado através fatores medidos dos campos quadrados.

Segundo Boyer e colaboradores (2001a), quando o formato do campo de radiação é irregular, o método de cálculo para obtenção de um fator mais preciso é menos trivial. Como a natureza física do espalhamento do colimador permanece inalterada quando um MLC é usado para dar forma ao campo de radiação, muitos métodos basearam-se em fontes de radiação extrafocal para o cálculo, podendo ser utilizados para estimar o fator espalhamento em campos irregulares.

Os métodos que podem ser devidamente aplicados para calcular o fator espalhamento de campos irregulares devidos ao formato das lâminas MLC, são aqueles baseados em algoritmos de convolução ou superposição (Ahnesjo e colaboradores (1992); Sharpe e Jaffray (1995)). Supondo que a fonte extrafocal seja radialmente simétrica, métodos comumente usados para o cálculo do espalhamento em um objeto simulador, assim como o convencional método de Clarkson, podem ser utilizados.

Atualmente existem softwares comerciais de cálculo de dose independente (também chamado de cálculo independente de unidades monitoras). Alguns estão sendo desenvolvidos e utilizados em muitos centros como uma ferramenta do controle de 
qualidade, como descrito por Boyer e colaboradores (1999), Zhu e colaboradores (2003) e Xing e colaboradores (2000).

Alguns desses algoritmos de cálculos utilizam ou são baseados no método de integração de Clarkson, para definição do campo equivalente colimado e respectivo fator espalhamento.

\subsection{Calculo independente de unidade monitora para IMRT}

A verificação do calculo de unidades monitoras para IMRT é bastante complexo e quase impraticável. Convencionalmente, embora as doses no sistema de planejamento sejam calculadas como distribuições, as condições de normalização utilizadas pelo algoritmo podem ser relacionadas à calibração fornecendo as unidades monitoras. Em IMRT, como a intensidade do feixe é modulada, não é conveniente o cálculo manual das unidades monitoras, sendo mais prático o desenvolvimento de um programa computacional independente.

Nos últimos anos vem sendo estudado e desenvolvido alguns algoritmos de cálculo de unidade monitora independentes do sistema de planejamento comercial para verificação do plano de tratamento previamente. Boyer e colaboradores (1999) apresenta em seu trabalho algumas considerações teóricas importantes para desenvolvimento de um método de verificação independente de unidade monitora.

Estes sistemas devem ser considerados como controle de qualidade quantitativo para verificação adicional de qualquer software comercial utilizado como sistema de planejamento de IMRT.

Dos resultados obtidos por estudos previamente publicados por diversos autores, conclui-se que o algoritmo de cálculo de dose independente pode ser utilizado como uma ferramenta para o controle de qualidade dos tratamentos de IMRT, aumentando o nível de acurácia da dose administrada no paciente.

Existem diferentes metodologias desenvolvidas para o cálculo independente de unidades monitoras, e independente da tecnologia empregada, deve-se comissionar e testar cuidadosamente qualquer software antes da sua utilização. Estes testes são importantes para se conhecer a acurácia e as limitações do sistema de verificação e estabelecimento dos níveis de ação. 


\section{MATERIAIS E MÉTODOS}

Após o comissionamento da técnica de IMRT é essencial que seja desenvolvido um programa de controle de qualidade abrangente para manter a acurácia, tolerância e especificações originais do sistema. Este programa deve incluir verificações da radiação liberada pelo acelerador assim como testes das lâminas. Em geral segue-se a recomendação de protocolos de controle de qualidade internacionais.

Por causa da complexidade dos campos de IMRT e da dificuldade de verificação da dose de tratamento por cálculos manuais, como é feito nos planos convencionais, é recomendado que, além dos testes periódicos do sistema, faça-se uma verificação do plano antes do tratamento de forma individualizada.

Recomenda-se realizar uma análise independente para todos os planos de tratamento de IMRT, principalmente nos primeiros anos de utilização da técnica. Em geral, a verificação dosimétrica é feita através da transferência de cada plano para um objeto simulador, no qual é realizado o cálculo. O objeto simulador deve possuir um ou mais dosímetros e ser irradiado com os campos de IMRT planejados para o paciente. Desta forma, a dose medida pode ser comparada com a dose planejada.

Recentemente vem sendo desenvolvidos sistemas independentes de cálculo de unidade monitora, para verificação garantido maior acurácia e qualidade. Esses programas simplificam a avaliação de dose de IMRT.

\subsection{Calculo independente de unidade monitora para IMRT}

O cálculo de unidades monitoras (UM) para IMRT foi desenvolvido através de uma analogia ao cálculo UM em teleterapia convencional, como sugerido por Kung e colaboradores (2000).

Na radioterapia conformada tridimensional (RTC-3D), o plano de tratamento é gerado através de um sistema de planejamento computadorizado (TPS - Treatment Planning System) e, antes da liberação do tratamento, é comum a verificação do cálculo das unidades monitoras.

As informações necessárias para verificação do cálculo das unidades monitoras em RTC3D são as seguintes: energia do feixe; tamanho do campo de radiação; campo 
quadrado equivalente para a área de tratamento; profundidade de penetração no tecido num determinado ponto (ponto de normalização da dose) para cada campo; acessórios (bandeja, filtro, etc.); e porcentagem da contribuição de dose para cada campo no ponto escolhido.

Com estes dados, pode-se obter a unidade monitora de cada campo e comparála com a dose calculada pelo sistema de planejamento. Este processo é utilizado para verificar a acurácia do algoritmo de cálculo de dose do sistema de planejamento para um único ponto no volume de tratamento e assumindo meio homogêneo e equivalente à água, Podgorsak e colaboradores (2005).

Em radioterapia com IMRT, o plano também é gerado através de um sistema de planejamento, habilitado para a técnica. Por analogia, existe a possibilidade de um algoritmo independente para verificação da unidade monitora ou percentual de dose para o tratamento com IMRT. A partir do sistema de planejamento, podem-se obter algumas informações importantes para cada campo de IMRT, tais como, por exemplo: arquivos SMLC contendo as posições das lâminas durante o tratamento; tamanho de campo; unidade monitora e profundidade até o ponto de interesse. Usando essas informações podese calcular a contribuição de dose por cada campo de tratamento em um determinado ponto no tecido, em geral o isocentro. A soma dessas doses por campo deve estar de acordo com a dose calculada pelo sistema de planejamento.

O documento publicado em 1997 pela ESTRO (Booklet n³) descreve todas as medidas e cálculos necessários para a determinação da dose absorvida por unidade monitora (aceleradores lineares) ao longo do raio central. Estas recomendações foram feitas para técnicas convencionais, e são utilizadas como base para o cálculo de unidades monitoras para radioterapia com IMRT.

Para o desenvolvimento e teste do sistema de cálculo independente, utilizou-se quatro planos de tratamento gerados a partir do software Eclipse ${ }^{\circledR}$ V.8.6 (Varian Medical System, Palo Alto, CA), cujo algoritmo de cálculo de dose utilizado foi o PBC.

Utilizou-se também as informações do sistema de planejamento (arquivos SMLC, tamanho de campo, unidade monitora por campo, profundidade do isocentro ao longo do eixo central para cada campo), além dos dados dosimétricos de implementação do software comercial e do equipamento utilizado acelerador linear Varian 21iX, entre este dados temos: relação tecido máximo (TMR); relação espalhamento máximo (SMR); fator 
espalhamento pico (PSF); fator colimador (CF); e fator de transmissão das lâminas. Devemos também considerar a caracterização de penumbra na região das bordas do MLC.

A caracterização do fator de transmissão das lâminas de MLC no comissionamento do sistema de planejamento é mais importante para IMRT que para técnica RTC-3D porque as lâminas colimam parte da área de tratamento por uma fração de tempo maior durante a administração da dose.

A maioria dos sistemas de planejamento utiliza um valor de transmissão médio, portanto, o sistema de medida (filme ou câmara de ionização) deve abranger uma região de medida de forma que a transmissão através do corpo da lâmina e entre duas lâminas adjacentes seja adequadamente quantificada.

Segundo Boyer e colaboradores (2001a), o fator transmissão pode ser medido de diversas formas, como por exemplo, faz-se uma medida com um campo de $10 \mathrm{~cm} \mathrm{x}$ $10 \mathrm{~cm}$ e as lâminas retraídas, de forma que o campo fique aberto, obtendo assim uma dose de referência, e então efetua-se a mesma medida mas com as lâminas fechadas sobre o campo. Através de um sistema calibrado de escaneamento e avaliação dosimétrica do filme, é permitido saber as variações de dose nas regiões sob as lâminas e quantificar a transmissão inter e intra-lâmina. Alternativamente, uma câmara de ionização pode ser utilizada para medir a transmissão média sob diversas lâminas adjacentes.

Estas medidas de transmissão inter e intra-lâmina podem ser executadas em diversas angulações de gantry, para que seja levada em conta o efeito da gravidade sob as lâminas.

O campo de radiação não pode ser completamente bloqueado pelo fechamento de um par de lâminas de MLC, especialmente quando lâminas de bordas arredondadas são utilizadas. Para essas áreas, chamadas de região de penumbra, deve ser medida com um detector de alta resolução (filmes e diodos permitem um levantamento mais preciso da penumbra).

O sistema de colimadores MLC da Varian, utilizado neste trabalho, tem bordas arredondadas e a contribuição de espalhamento é em torno de $20 \%$, segundo Chui e colaboradores (2001), e pode chegar até 50\% conforme a publicação de Boyer e colaboradores (2001a). 
Cada plano de tratamento utilizado no estudo é composto por 5 campos de angulações distintas e com contribuição homogênea de dose no volume alvo, como mostrado na figura 8. O isocentro foi considerado como ponto de referência para comparação da dose calculada e da dose obtida através do sistema de planejamento. A energia dos feixes utilizados foi de 6MV. O Método de entrega de dose escolhido foi o step and shoot (SMLC), no qual a dose por campo é entregue em forma de diversos subcampos estáticos.

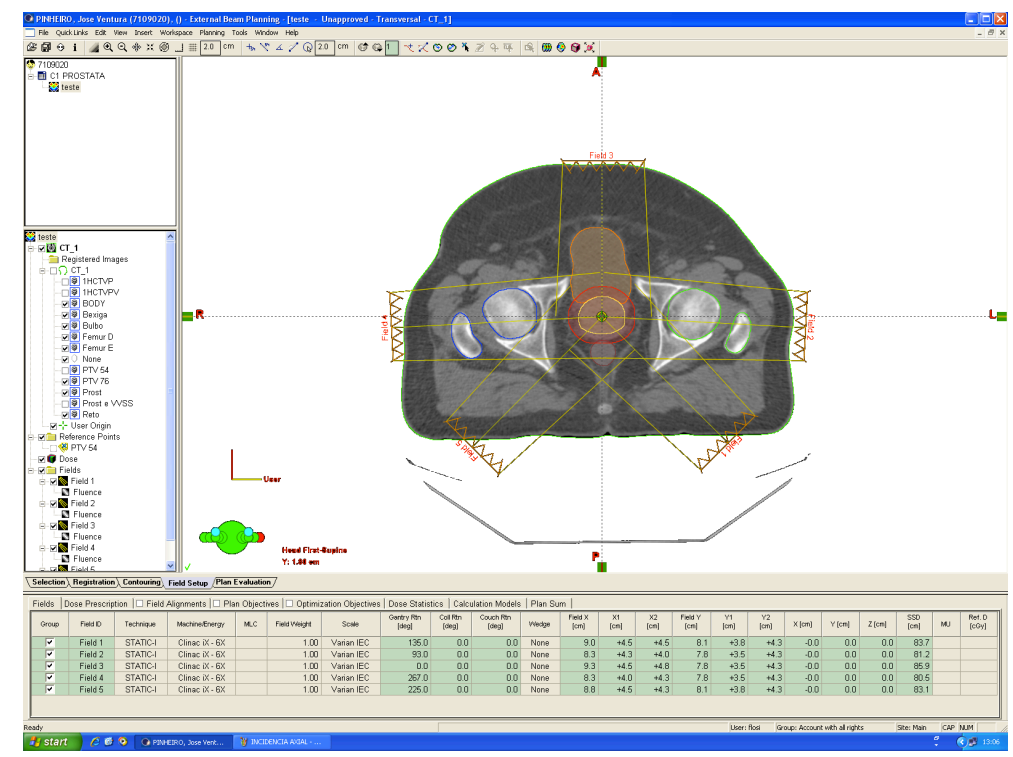

Figura 8 - Distribuição da incidência dos 5 campos modulados utilizados em cada plano de tratamento: $135^{\circ}, 93^{\circ}, 0^{\circ}$, $267^{\circ}$ e $225^{\circ}$.

Os aceleradores lineares utilizados em radioterapia, possuem em geral uma abertura de campo máxima de $40 \mathrm{~cm}$ x $40 \mathrm{~cm}$, e o conjunto de lâminas MLC devem cobrir toda esta extensão. O equipamento utilizado para o estudo possui 120 lâminas no total, sendo 60 do grupo A (lado esquerdo do campo) e 60 do grupo B (lado direito do campo), sendo que os $20 \mathrm{~cm}$ centrais do campo são cobertos por 40 lâminas de cada grupo com $0,5 \mathrm{~cm}$ de largura e os $10 \mathrm{~cm}$ das extremidades superior e inferior por lâminas de $1,0 \mathrm{~cm}$ de largura, como ilustrado na Figura 9. 


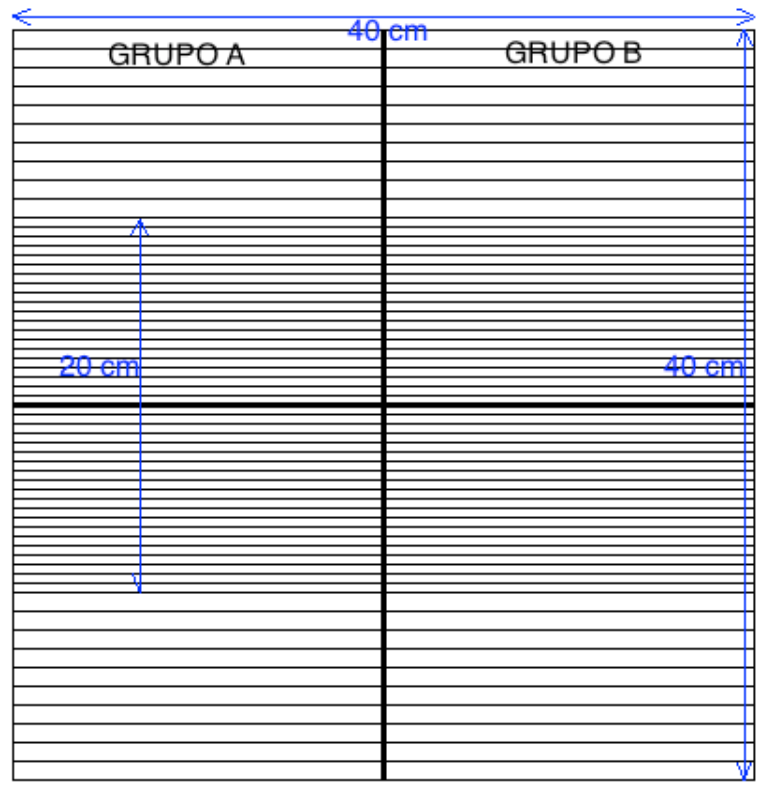

Figura 9 - Esquema ilustrativo do colimador de múltiplas lâminas (MLC) de 120 lâminas utilizado no estudo.

Para cada campo de cada plano existem em média 20 subcampos, como mostrado na Figura: a fluência do campo de tratamento é resultado da somatória de cada subcampo de radiação, como mostrado na Figura 11 para um feixe de $6 \mathrm{MV}$. O método de cálculo e entrega de dose step and shoot fornece informações da configuração das lâminas e respectivas UM para cada um deles. Para organizar os dados, os arquivos SMLC foram transformados maunualmente em planilhas do Excel (.xls). No Apêndice A são mostrados os dados de um subcampo, ainda não trabalhados.
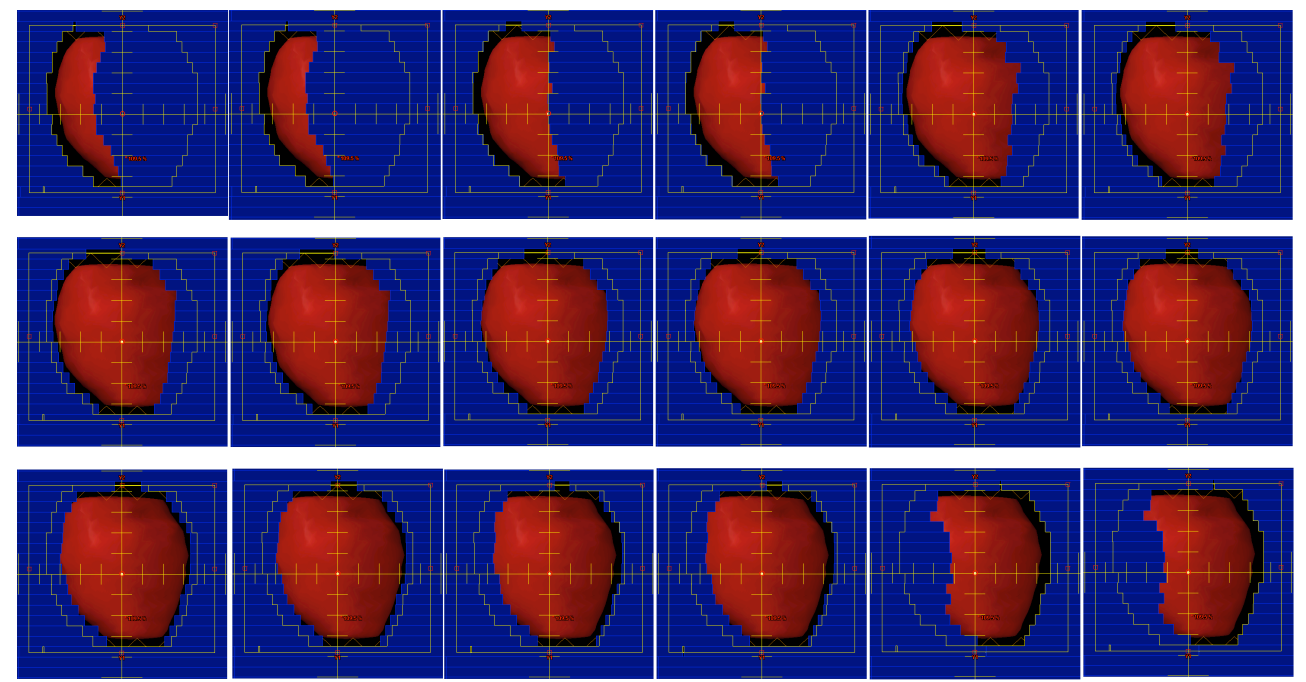

Figura 10 - Campo modulado como resultado da subdivisão em 18 subcampos conformados com diferentes formas de arranjo do MLC. 


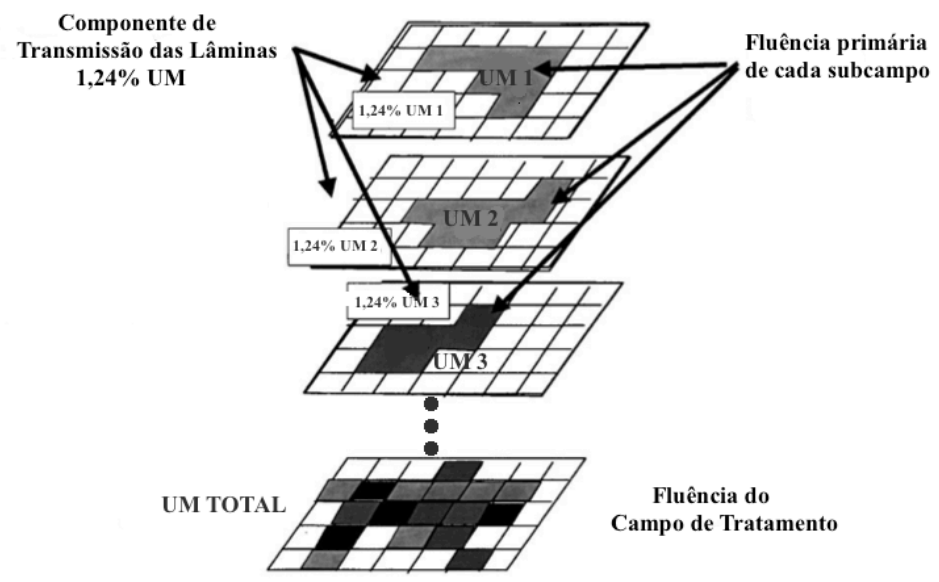

Figura 11 -Fluência modulada do campo de tratamento mostrado como somatória de fluências planas de cada subcampo e da componente de transmissão através das lâminas, para um feixe de 6MV. Figura modificada de Kung e colaborares (2000).

Os arquivos SMLC, como mostrados no apêndice A, tem a informação da posição que cada lâmina ocupa em relação ao eixo central do campo de radiação. Se a extremidade da lâmina estiver antes do eixo central a posição é positiva, caso a lâmina esteja além do eixo central a posição é negativa, dada em centímetros. Desta forma, podese reproduzir o formato de cada subcampo e seu respectivo campo colimado equivalente através do método de Clarkson, descrito a seguir. Além disso, os arquivos também nos fornecem o índice de cada subcampo, ou seja, a fração de UM do campo total. Para o subcampo da Tabela 1 o índice é de 0,0625 , ou seja, 6,25\% da UM total do campo em estudo.

\subsection{Desenvolvimento da metodologia}

O objetivo deste trabalho é desenvolver um método de cálculo independente para encontrar a dose em determinado ponto no tecido uma vez conhecidas a energia do feixe, as dimensões do campo, os arquivos SMLC e a unidade monitora para cada campo e respectivo subcampo do plano de tratamento.

A primeira parte da solução deste problema consiste no cálculo de dose no tecido ao longo do raio central do campo de radiação para cada subcampo de tratamento, e para realização deste cálculo, é necessário obter o tamanho do campo equivalente colimado, através do método de Clarkson, para cada subcampo. Este cálculo foi realizado 
manualmente para todos os subcampos de cada campo do plano de tratamento. Assume-se que a superfície do paciente seja plana e o tecido seja homogêneo.

O método de Clarkson é baseado no princípio de que a componente de espalhamento no ponto de cálculo de dose, que depende do tamanho e do formato do campo, pode ser calculado separadamente do componente primário que é independente do tamanho e formato do campo. Duas grandezas especiais são introduzidas no cálculo de dose, a relação tecido - objeto simulador para um campo de tamanho zero, $\operatorname{TPR}(0)$, e a relação espalhamento - objeto simulador para um campo de tamanho zero, $\operatorname{SPR}(0)$.

Para cada subcampo, a componente da dose espalhada no ponto de interesse, no isocentro, foi calculado dividindo o subcampo em setores angulares de $\Delta \theta=5^{\circ}$ para maior precisão. O número total de setores, $N$, é calculado pela seguinte equação:

$$
N=\frac{2 \pi}{\Delta \theta}=\frac{2 \pi}{5}
$$

A componente da dose espalhada para cada setor angular é obtido através da dose espalhada de um campo circular com raio $r$, igual ao raio do setor. E a componente de dose espalhada para cada campo irregular (subcampo) no isocentro, com profundidade $z$, é então calculada através da expressão:

$$
\operatorname{SPR}(z, \operatorname{subcampo})=\frac{1}{N} \sum_{i=1}^{N} \operatorname{SPR}\left(z, r_{i}\right)
$$

em que $\operatorname{SPR}\left(z, r_{i}\right)$ é a componente de dose espalhada para um campo circular de raio $\mathrm{r}_{\mathrm{i}}$, profundidade $z$ e $r_{i}$, é o raio do i-ésimo setor.

Finalmente, para obter a dose espalhada, a TPR do subcampo no ponto P é calculado da seguinte forma:

$$
\operatorname{TPR}\left(z, r_{i}\right)=\left[\operatorname{TPR}(z, 0)+\operatorname{SPR}\left(z, r_{i}\right)\right] \cdot \frac{s_{Q}(0)}{S_{Q}\left(r_{i}\right)}
$$

em que $S_{Q}\left(r_{i}\right)$ é o fator espalhamento - objeto simulador médio para o subcampo no ponto de interesse, isocentro, e $S_{Q}(0)$ é o fator espalhamento - objeto simulador para um campo de tamanho zero. 
A partir do termo $\operatorname{TPR}\left(z, r_{i}\right)$, pode-se encontrar o valor do campo equivalente colimado para cada subcampo na profundidade do isocentro. Os valores da $\operatorname{TPR}\left(z, r_{i}\right)$ e $\operatorname{SPR}\left(z, r_{i}\right)$ são tabelados.

Nos cálculos para verificação da dose por campo de tratamento, incluiu-se também a transmissão do campo de radiação através das lâminas (MLC), para regiões dentro do campo de radiação bloqueadas pelas lâminas, ou seja, para os subcampos cujo ponto de referência (isocentro) estava colimado com uma margem de $3 \mathrm{~mm}$ da borda das lâminas, considerou-se duas a dose proveniente da transmissão através do corpo das lâminas, ou seja, 1,24\% da UM por subcampo, para um feixe com energia de $6 \mathrm{MV}$.

Para os subcampos cujo isocentro não estava colimado, a dose foi calculada de forma convencional, ou seja, utilizou-se o método de Clarkson para obtenção do campo equivalente colimado (CEC) e os dados do TPS para cálculo da dose, através da equação abaixo.

$$
\text { Dose }=(U M / S C) \times T M R(C E C) \times F_{t} \times \frac{\% I S O}{100}
$$

em que, UM/SC é o valor da unidade monitora por subcampo; TMR(CEC) é a razão tecido máximo para o campo equivalente colimado; $F_{t}$ são todos os fatores adicionados os campo (bandeja, filtro, rendimento, etc.) e ISO é a isodose de normalização do plano de tratamento.

Nos casos em que o isocentro estava parcialmente colimado pelas lâminas, ou seja, o limite da borda das lâminas estava a uma distância inferior a $3 \mathrm{~mm}$, considerou-se que contribuição de dose proveniente da transmissão das bordas lâminas, 28\% da UM por subcampo. Nestes casos, o ponto de cálculo de dose encontra-se numa região crítica, chamada região de penumbra, na qual ao gradiente de dose é alto e medidas dosimétricas cuidadosas são necessárias no caso de IMRT. O valor utilizado foi encontrado através de medidas utilizando um conjunto de câmaras de ionização e objeto simulador, e também análise através de filme radiográfico e como consideramos uma região de $6 \mathrm{~mm}$, sendo $3 \mathrm{~mm}$ além do limite das lâminas e $3 \mathrm{~mm}$ antes desse limite, não sendo possível obter um valor único devido ao alto gradiente, o valor utilizado é um valor médio. 
A Figura 12 mostra o fluxograma do algoritmo de cálculo. A dose total é comparada com a dose no isocentro calculada pelo sistema de planejamento Eclipse ${ }^{\circledR}$.

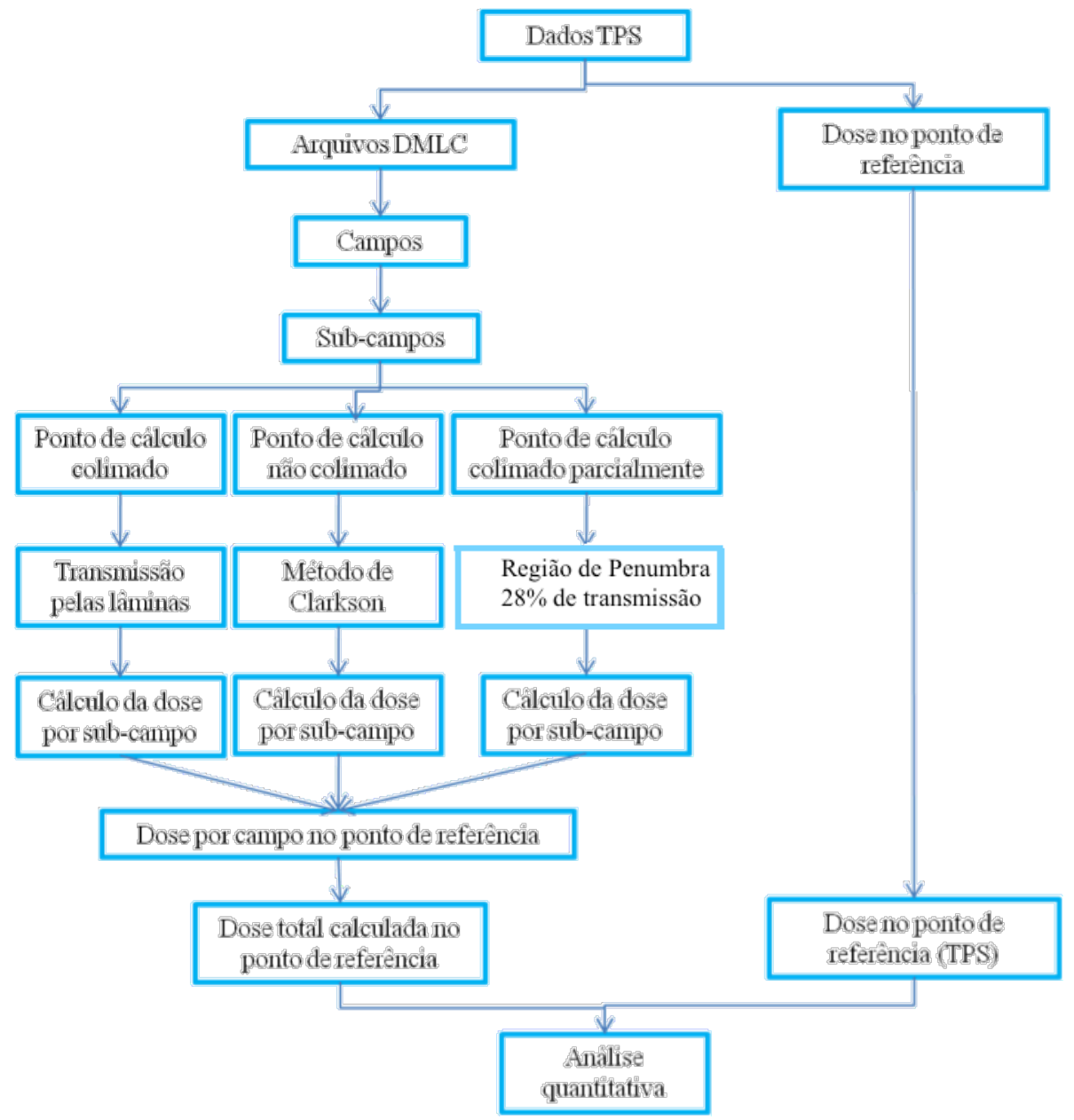

Figura 12 - Fluxograma do algoritmo de cálculo desenvolvido

A diferença relativa entre os valores de dose total calculados de forma independente $\left(D_{S I}\right)$ e os valores calculados pelo sistema de planejamento $\left(D_{T P S}\right)$ foi calculada para cada plano utilizando a relação a seguir:

$$
D R=\left|\frac{D_{S I}-D_{T P S}}{D_{T P S}}\right| \cdot 100
$$




\section{RESULTADOS: ANÁLISE E DISCUSSÃO}

A partir dos dados gerados pelo sistema de planejamento do plano de tratamento otimizado e calculado para cada caso em estudo, foi organizada uma planilha separando previamente os elementos, energia do feixe de fótons, campo quadrado equivalente como mostrado na Tabela 1. Nesta tabela são mostrados os dados do caso $1 \mathrm{em}$ estudo, derivado de um plano de tratamento de neoplasia de próstata, em que foram utilizados uma distribuição de 5 campos coplanares com IMRT e energia dos fótons de $6 \mathrm{MV}$

Tabela 1 - Tabela de dados gerados a partir do sistema de planejamento para IMRT - Caso 1.

\begin{tabular}{|l|c|c|c|c|c|}
\hline Campo & $\mathbf{1}$ & $\mathbf{2}$ & $\mathbf{3}$ & $\mathbf{4}$ & $\mathbf{5}$ \\
\hline Energia (MV) & 6 & 6 & 6 & 6 & 6 \\
\hline Campo quadrado equivalente & 10,2 & 9,3 & 10,5 & 9,3 & 10,6 \\
\hline Arquivo SMLC &. $\mathrm{mlc}$ &. $\mathrm{mlc}$ &. $\mathrm{mlc}$ &. $\mathrm{mlc}$ &. $\mathrm{mlc}$ \\
\hline Profundidade & 14,1 & 17,5 & 9,8 & 18,1 & 14,4 \\
\hline UM Total / Campo & 73 & 85 & 76 & 83 & 71 \\
\hline Dose / Campo D $(c G y)$ & 38,4 & 41,7 & 46,4 & 40,0 & 37,1 \\
\hline
\end{tabular}

No exemplo da Tabela 1 estão expressos os valores gerados pelo sistema de planejamento para cada campo de tratamento e as informações mais importantes para o cálculo da dose de forma independente, tais como: energia do feixe de fótons utilizada; profundidade de penetração do feixe de radiação no tecido no ponto de interesse (isocentro); campo quadrado equivalente, parâmetro importante para obtenção do fator rendimento e TMR; unidade monitora por campo de tratamento; arquivos SMLC com a informação do formato e da fração de unidade monitora para cada subcampo; e dose entregue por cada campo ao ponto de interesse (isocentro) em cGy.

Para o cálculo da dose entregue por cada campo de tratamento e posterior comparação com o valor obtido pelo sistema de planejamento, é necessária uma análise 
individual de cada campo e seus respectivos subcampos, uma vez que cada subcampo contribui de forma não homogênea para a dose no isocentro.

A avaliação de forma individualizada para cada campo foi feita com a organização de uma nova planilha de forma que os subcampos pudessem ser analisados separadamente, uma vez que os arquivos das posições das lâminas foram calculados pelo método step and shoot (SMLC) que contém informações sobre as unidades monitoras e formatos estáticos para cada subcampo. Um exemplo desta planilha está explicitada na Tabela 2 para um dos campos calculados.

Tabela 2 - Planilha contendo dados individualizados para um campo e seus respectivos subcampos. No exemplo desta tabela, os dados são referentes ao campo 3 do caso 1 em estudo.

\begin{tabular}{|c|c|c|c|c|c|c|c|c|c|c|}
\hline \multicolumn{11}{|c|}{ Caso 1 - Campo 3} \\
\hline Sub-campo & 1 & 2 & 3 & 4 & 5 & 6 & 7 & 8 & 9 & 10 \\
\hline Formato MLC & +3 & 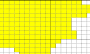 & & & & & & & & \\
\hline $\begin{array}{l}\text { Campo quadrado } \\
\text { equivalente colimado }\end{array}$ & 4,2 & 8,3 & 9,1 & 9,3 & 9,4 & 9,4 & 9,2 & 8,7 & 7,9 & 3,9 \\
\hline Prof. de cálculo & 9,8 & 9,8 & 9,8 & 9,8 & 9,8 & 9,8 & 9,8 & 9,8 & 9,8 & 9,8 \\
\hline$U M /$ Sub-campo & 7,6 & 7,6 & 7,6 & 7,6 & 7,6 & 7,6 & 7,6 & 7,6 & 7,6 & 7,6 \\
\hline$T M R$ & 0,729 & 0,770 & 0,776 & 0,777 & 0,777 & 0,777 & 0,776 & 0,773 & 0,766 & 0,726 \\
\hline Fator Rendimento & 0,982 & 0,997 & 0,999 & 1,000 & 1,000 & 1,000 & 1,000 & 0,998 & 0,996 & 0,981 \\
\hline $\begin{array}{l}\text { Dose Calculada / Sub- } \\
\text { campo }(c G y)-D_{S C}\end{array}$ & 0,09 & 5,94 & 6,00 & 6,01 & 6,01 & 6,01 & 5,97 & 5,90 & 2,25 & 0,09 \\
\hline $\begin{array}{l}\text { Dose Total Calculada / } \\
\text { Campo }(c G y)-D_{C}\end{array}$ & \multicolumn{10}{|c|}{44,27} \\
\hline
\end{tabular}

$\mathrm{Na}$ Tabela 2 são explicitados os dados específicos de cada campo e seus respectivos subcampos a partir dos arquivos SMLC: incluindo o seu formato de colimação das lâminas, importante para cálculo fator rendimento e TMR (incluídos na planilha); profundidade do ponto de cálculo; fração de unidade monitora por subcampo. Para o cálculo da dose foi utilizada a relação apresentada na Equação 8 em todos os casos, porém nos quais houve colimação do isocentro, como nos subcampos 1 e 10 da Tabela 2, foram 
considerados que apenas $1,24 \%$ da unidade monitora por subcampo contribuiu para dose no ponto de interesse, devido a transmissão através das lâminas, e nos casos de colimação parcial do isocentro, como no subcampo 9 da Tabela 2, considerou-se $28 \%$ da unidade monitora por subcampo contribuindo para dose no isocentro.

Com os valores das contribuições de dose no isocentro por subcampo $\left(D_{S C}\right)$ determinou-se a dose por campo $\left(D_{C}\right)$ e a respectiva dose total entregue no isocentro $\left(D_{\text {Total }}\right)$ a partir das relações abaixo, para cada caso em estudo:

$$
\begin{aligned}
& D_{C}=\sum_{i=1}^{n} D_{S C}^{i} \\
& D_{\text {Total }}=\sum_{i=1}^{5} D_{C}^{i}
\end{aligned}
$$

Com os valores das doses por subcampo $\left(D_{S C}\right)$ e da respectiva dose por campo $\left(D_{C}\right)$ calculadas de forma independente, pode-se comparar com as doses calculadas pelo sistema de planejamento.

Foi analisado um total de 20 campos modulados de tratamentos, os desvios relativos foram dispostos num histograma para análise da distribuição.

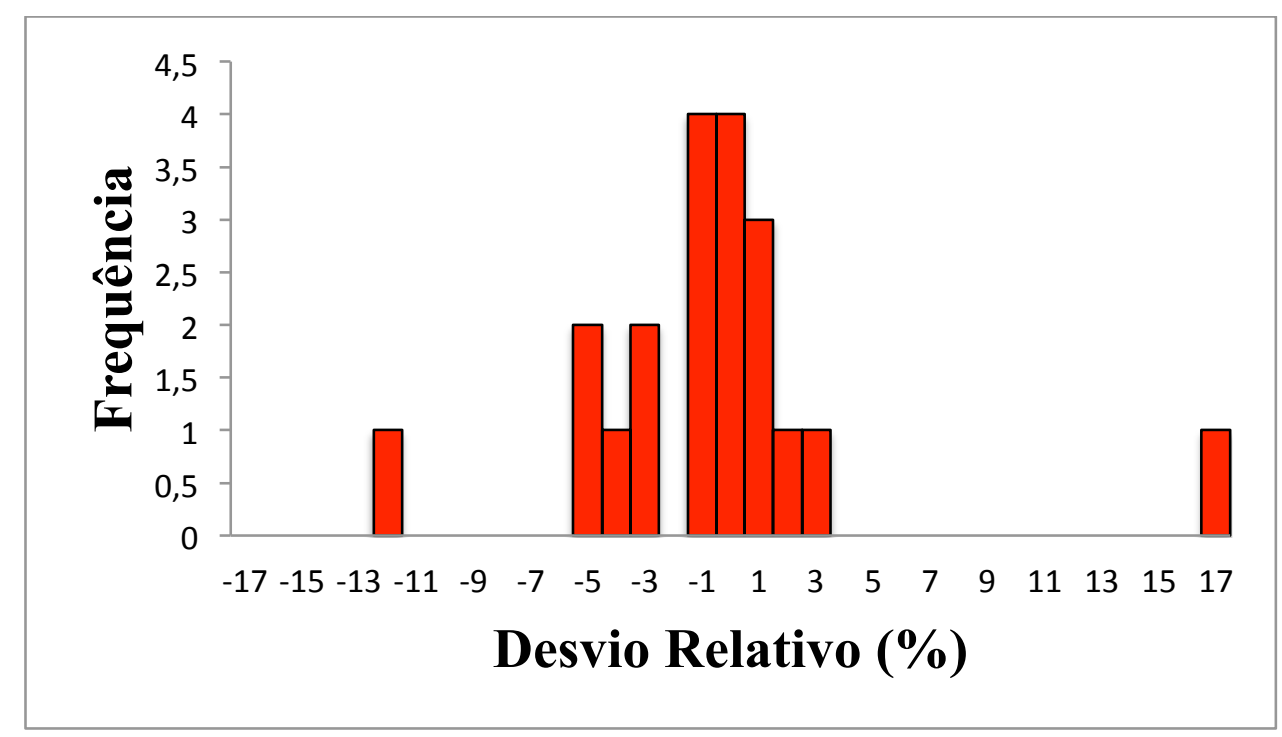

Figura 13 - Histograma do desvio relativo de cada campo, num total de 20 campos calculados.

A análise do histograma do desvio relativo dos campos, calculado através da equação 15 , mostra que o valor da dose calculado pelo sistema independente de 18 dos 20 
campos teve um desvio relativo menor que $5 \%$ do valor calculado pelo sistema de planejamento. Apenas dois campos apresentaram uma discrepância maior, sendo os dois pertencentes ao mesmo plano de tratamento, o que não interferiu no valor total de dose calculada no isocentro plano em estudo. Estes dois campos que apresentaram desvio relativo da dose maior que $5 \%(D R=-12,5 \%$ e $D R=17 \%)$ faziam parte de um plano mais otimizados que os demais.

Quando um plano de tratamento precisa ser mais otimizado que o convencional, devido a características anatômicas do paciente, a fluência de dose de cada campo torna-se um pouco mais complexa apresentando regiões com gradiente de dose mais elevado, aumentando assim o número de subcampos necessários para obtenção desta fluência de dose e, consequentemente, reduzindo a quantidade de unidades monitoras por subcampo. Nestes casos, devido a redução na quantidade de unidades monitoras por subcampo (menores que 10), uma diferença unitária no resultado encontrado pode significar um desvio relativo grande.

Neste trabalho foi observado que os valores calculados de forma independente, na maioria dos casos, foram menores que os valores calculados através do sistema de planejamento comercial, podendo ser justificado pelo fato de que não foi levado em conta a contribuição de dose espalhada pelas lâminas de MLC.

A verificação da unidade monitora é realizada pelo físico médico e serve para validar as características do equipamento utilizado para o tratamento implementadas no sistema de planejamento e detectar possíveis fontes de erro, se tornando um importante elemento no programa de controle de qualidade visando um tratamento seguro e acurado do paciente oncológico. Em geral, este tipo de verificação deve ser realizado prétratamento em conjunto com uma série de testes dosimétricos com câmaras de ionização, diodos, dosímetros termoluminescentes, ou através de filmes fotográficos e arranjos lineares de detetores, e não de forma individualizada.

Mesmo que o sistema de planejamento possua mais que um modelo de cálculo implementado, a utilização de um programa de independente por outro sistema é fortemente recomendado por Stern e colaboradores no Task Group 114 (2011), pois a utilização do mesmo sistema pode esconder parâmetros ou fonte de erros comuns a ambos modelamentos. 


\subsection{Estimativas das incertezas}

A incerteza do cálculo do sistema de planejamento é uma combinação da própria incerteza de cálculo do algoritmo e das incertezas referentes aos valores medidos que são inseridos como base para o algoritmo. Dentre elas temos a incerteza de medida do conjunto dosimétrico, composto por eletrômetro e câmara de ionização, e da incerteza da calibração do conjunto dosimétrico num laboratório padrão em termos de dose absorvida na água, incerteza esta declarada no certificado de calibração emitido pelo laboratório de calibração.

Para a aquisição dos dados dosimétricos foi utilizada uma câmara de ionização cuja precisão de medida é cerca de $\pm 0,5 \%$ na corrente medida. O conjunto objeto simulador e câmara de ionização possui uma resolução em termos de posicionamento de $\pm 1,0 \mathrm{~mm}$.

Para estimativa da incerteza na determinação da dose absorvida na água sob condições de referência, segundo o protocolo da Agência Internacional de Energia Atômica, TRS-398 (2000), a incerteza nas diferentes grandezas físicas e procedimentos que contribuem para determinação da dose podem ser divididos em duas etapas.

Etapa 1: considera incertezas da calibração do dosímetro de referência do usuário em termos do fator de calibração dos laboratórios padrões.

Etapa 2: trata a calibração do feixe do usuário e inclui incertezas associadas com as medidas no ponto de referência em um objeto simulador de água. A etapa 2 inclui a incerteza do valor do fator de correção da qualidade do feixe de radiação.

Combinando as incertezas nas várias etapas obtém-se a incerteza padrão combinada para a determinação da dose absorvida na água no ponto de referência. 
Tabela 3 - Detalhamento do cálculo das incertezas.

\begin{tabular}{|l|c|c|}
\hline Componente de incerteza & Incerteza tipo A & Incerteza tipo B \\
\hline $\begin{array}{l}\text { Fator de calibração, } N_{D, w} \text { fornecido pelo } \\
\text { LCI/IPEN }\end{array}$ & & $1,4 \%$ \\
\hline Leitura associada ao conjunto dosimétrico & $0,5 \%$ & \\
\hline $\begin{array}{l}\text { Posicionamento do objeto simulador para uma } \\
\text { SSD }=100 \text { cm }\end{array}$ & $0,2 \%$ & \\
\hline $\begin{array}{l}\text { Determinação da qualidade do feixe }- \text { TPR } 20,10 \\
\text { Determinação do fator de qualidade da câmara } \\
\text { de ionização }-K_{Q, Q 0}\end{array}$ & $0,5 \%$ & $2,0 \%$ \\
\hline $\begin{array}{l}\text { Incerteza combinada }(k=2) \\
\text { Incerteza total combinada }(k=2)\end{array}$ & $0,7 \%$ & $2,4 \%$ \\
\hline
\end{tabular}

$\mathrm{Na}$ Tabela 3 pode-se ver de forma detalhada as componentes das incertezas envolvidas na calibração do equipamento, na qual incertezas tipo A são aquelas avaliadas por métodos estatísticos e incertezas tipo B são avaliadas por outros meios.

As doses encontradas e as suas respectivas incertezas estão explicitadas na Tabela 4.

Tabela 4 - Dose calculada independente com sua respectiva incerteza.

\begin{tabular}{|c|c|c|c|}
\hline Caso & $\begin{array}{c}\text { Dose calculada independente } \\
(\mathbf{k}=\mathbf{2})\end{array}$ & $\begin{array}{c}\text { Dose TPS } \\
(\mathbf{\sigma}=\mathbf{2 \%})\end{array}$ & DR (\%) \\
\hline 1 & $(200,4 \pm 5,0) \mathrm{cGy}$ & $(203,7 \pm 4,1) \mathrm{cGy}$ & 1,6 \\
\hline 2 & $(200,4 \pm 5,0) \mathrm{cGy}$ & $(201,7 \pm 4,0) \mathrm{cGy}$ & 0,6 \\
\hline 3 & $(205,7 \pm 5,1) \mathrm{cGy}$ & $(209,4 \pm 4,2) \mathrm{cGy}$ & 1,8 \\
\hline 4 & $(210,7 \pm 5,3) \mathrm{cGy}$ & $(208,5 \pm 4,2) \mathrm{cGy}$ & 1,1 \\
\hline
\end{tabular}

Conforme visto na tabela 4, aumentando-se o intervalo de confiança para 2 incertezas padrão (nível de confiança de 95\%, usualmente adotado nos laboratórios padrões e na dosimetria em radioterapia), todos os valores calculados de forma independente estão bem próximos com os valores calculados através do sistema de planejamento comercial. 
Os sistemas de planejamento comerciais não costumam divulgar detalhes dos mecanismos de cálculo de dose para os usuários, não sendo possível estimar precisamente o valor da incerteza do cálculo computacional para uma análise estatística completa. Estima-se que esta incerteza para um algoritmo do tipo Pencil Beam Convolution esteja entre 2 e $3 \%$, para cálculos que não incluem correção de heterogeneidade dos tecidos. 


\section{CONCLUSÕES}

A qualidade de um tratamento de radioterapia com IMRT é resultado de muitos fatores, tais como a experiência da equipe técnica, a escolha ideal do programa de controle de qualidade do equipamento e de cada plano individualmente, a escolha correta dos níveis de aceitação e a duplicidade na verificação dos dados nas várias etapas do tratamento, entre outros.

O cálculo das unidades monitoras e/ou dose de forma independente complementam a avaliação do plano de forma quantitativa, tornando-se assim uma ferramenta importante de verificação da qualidade do plano de tratamento, uma vez que erros sistemáticos no sistema de planejamento são muito difíceis e trabalhosos para serem detectados. Uma ação prática e eficiente seria a utilização desses algoritmos na avaliação da qualidade do cálculo e detecção de possíveis erros.

Neste trabalho foi apresentado o desenvolvimento de um algoritmo de cálculo independente de unidades monitoras como uma nova ferramenta para garantir ainda mais a qualidade e acurácia dos tratamentos. Os resultados apresentaram uma concordância dentro de $\pm 1,8 \%$ com os valores calculados pelo sistema de planejamento comercial, estando dentro do valor adotado como referência por órgãos e comissões internacionais.

O algoritmo desenvolvido deve ser implementado de forma segura após a realização de diversos testes associados aos testes dosimétricos comparativos já empregados na rotina de controle de qualidade de IMRT, e ainda pode ser melhorado através da inclusão da contribuição da dose espalhada pelo próprio MLC.

Após diversos testes e conhecendo o sistema, com os níveis de ação devidamente estabelecidos, a verificação independente da unidade monitora para planos de tratamento de IMRT se tornará uma ferramenta efetiva e eficiente no controle de qualidade que ajuda a identificar e reduzir possíveis erros de tratamento em radioterapia.

Como contribuição original deste trabalho assegura-se aos serviços de Radioterapia que não dispõem de recursos econômicos para adquirirem softwares já comercialmente disponíveis para o calculo independente da unidade monitora, a utilização 
a metodologia desenvolvida neste trabalho para desenvolvimento de ferramenta de controle de qualidade em tratamentos com IMRT. 


\section{APÊNDICE A - Arquivo contendo o posicionamento das lâminas de MLC.}

Tabela A - Informação do posicionamento de cada lâmina para um subcampo, por determinada fração de UM

\begin{tabular}{|c|c|c|c|}
\hline $\begin{array}{l}\text { Campo }=1-\text { POST.0 } \\
\text { Índice }=0.0000 \\
\text { Colimador }=0.0\end{array}$ & & $\begin{array}{l}\text { Campo }=1-\text { POST. } 1 \\
\text { Índice }=0.0625 \\
\text { Colimador }=0.0\end{array}$ & \\
\hline Lâmina $1 \mathrm{~A}=-5.300$ & Lâmina 1B = 5.300 & Lâmina $1 \mathrm{~A}=-5.300$ & Lâmina $1 \mathrm{~B}=5.300$ \\
\hline Lâmina $2 \mathrm{~A}=-5.300$ & Lâmina 2B $=5.300$ & Lâmina $2 \mathrm{~A}=-5.300$ & Lâmina 2B $=5.300$ \\
\hline Lâmina $3 A=-5.300$ & Lâmina 3B = 5.300 & Lâmina $3 A=-5.300$ & Lâmina 3B $=5.300$ \\
\hline Lâmina $4 \mathrm{~A}=-5.300$ & Lâmina 4B = 5.300 & Lâmina $4 \mathrm{~A}=-5.300$ & Lâmina 4B = 5.300 \\
\hline Lâmina $5 A=-5.300$ & Lâmina 5B = 5.300 & Lâmina $5 A=-5.300$ & Lâmina 5B = 5.300 \\
\hline Lâmina $6 A=-5.300$ & Lâmina 6B = 5.300 & Lâmina 6A = -5.300 & Lâmina 6B = 5.300 \\
\hline Lâmina $7 A=-5.300$ & Lâmina 7B = 5.300 & Lâmina 7A = -5.300 & Lâmina 7B = 5.300 \\
\hline Lâmina $8 \mathrm{~A}=-5.300$ & Lâmina 8B = 5.300 & Lâmina $8 \mathrm{~A}=-5.300$ & Lâmina 8B = 5.300 \\
\hline Lâmina $9 \mathrm{~A}=-5.300$ & Lâmina 9B = 5.300 & Lâmina 9A $=-5.300$ & Lâmina 9B = 5.300 \\
\hline Lâmina $10 \mathrm{~A}=-5.300$ & Lâmina $10 \mathrm{~B}=5.300$ & Lâmina $10 \mathrm{~A}=-5.300$ & Lâmina $10 \mathrm{~B}=5.300$ \\
\hline Lâmina $11 \mathrm{~A}=-5.300$ & Lâmina 11B = 5.300 & Lâmina $11 \mathrm{~A}=-5.300$ & Lâmina 11B = 5.300 \\
\hline Lâmina $12 \mathrm{~A}=-5.300$ & Lâmina 12B = 5.300 & Lâmina $12 \mathrm{~A}=-5.300$ & Lâmina $12 \mathrm{~B}=5.300$ \\
\hline Lâmina $13 A=-5.300$ & Lâmina 13B = 5.300 & Lâmina $13 \mathrm{~A}=-5.300$ & Lâmina $13 \mathrm{~B}=5.300$ \\
\hline Lâmina $14 \mathrm{~A}=-5.300$ & Lâmina 14B = 5.300 & Lâmina $14 \mathrm{~A}=-5.300$ & Lâmina 14B = 5.300 \\
\hline Lâmina $15 A=-5.300$ & Lâmina 15B = 5.300 & Lâmina $15 A=-5.300$ & Lâmina $15 \mathrm{~B}=5.300$ \\
\hline Lâmina $16 \mathrm{~A}=-5.300$ & Lâmina 16B = 5.300 & Lâmina $16 \mathrm{~A}=-5.300$ & Lâmina $16 \mathrm{~B}=5.300$ \\
\hline Lâmina $17 A=-5.300$ & Lâmina 17B = 5.300 & Lâmina $17 \mathrm{~A}=-5.300$ & Lâmina $17 \mathrm{~B}=5.300$ \\
\hline Lâmina $18 \mathrm{~A}=-5.300$ & Lâmina 18B = 5.300 & Lâmina $18 \mathrm{~A}=-5.300$ & Lâmina $18 \mathrm{~B}=5.300$ \\
\hline Lâmina $19 \mathrm{~A}=-5.300$ & Lâmina 19B = 5.300 & Lâmina $19 A=-5.300$ & Lâmina $19 B=5.300$ \\
\hline Lâmina $20 \mathrm{~A}=-5.300$ & Lâmina 20B = 5.300 & Lâmina $20 \mathrm{~A}=-5.300$ & Lâmina $20 \mathrm{~B}=5.300$ \\
\hline Lâmina $21 \mathrm{~A}=-5.300$ & Lâmina 21B = 5.300 & Lâmina $21 \mathrm{~A}=-5.300$ & Lâmina 21B $=5.300$ \\
\hline Lâmina $22 \mathrm{~A}=-5.300$ & Lâmina 22B = 5.300 & Lâmina $22 \mathrm{~A}=-5.300$ & Lâmina 22B $=5.300$ \\
\hline Lâmina $23 \mathrm{~A}=-1.730$ & Lâmina 23B = 1.890 & Lâmina $23 \mathrm{~A}=-1.730$ & Lâmina 23B = 1.890 \\
\hline Lâmina $24 \mathrm{~A}=-1.980$ & Lâmina 24B = 2.140 & Lâmina $24 \mathrm{~A}=-1.980$ & Lâmina 24B = 2.140 \\
\hline Lâmina $25 \mathrm{~A}=-1.510$ & Lâmina 25B = 2.390 & Lâmina $25 \mathrm{~A}=-1.510$ & Lâmina 25B = 2.390 \\
\hline Lâmina $26 \mathrm{~A}=-2.210$ & Lâmina 26B = 2.640 & Lâmina $26 \mathrm{~A}=-2.210$ & Lâmina 26B $=2.640$ \\
\hline
\end{tabular}




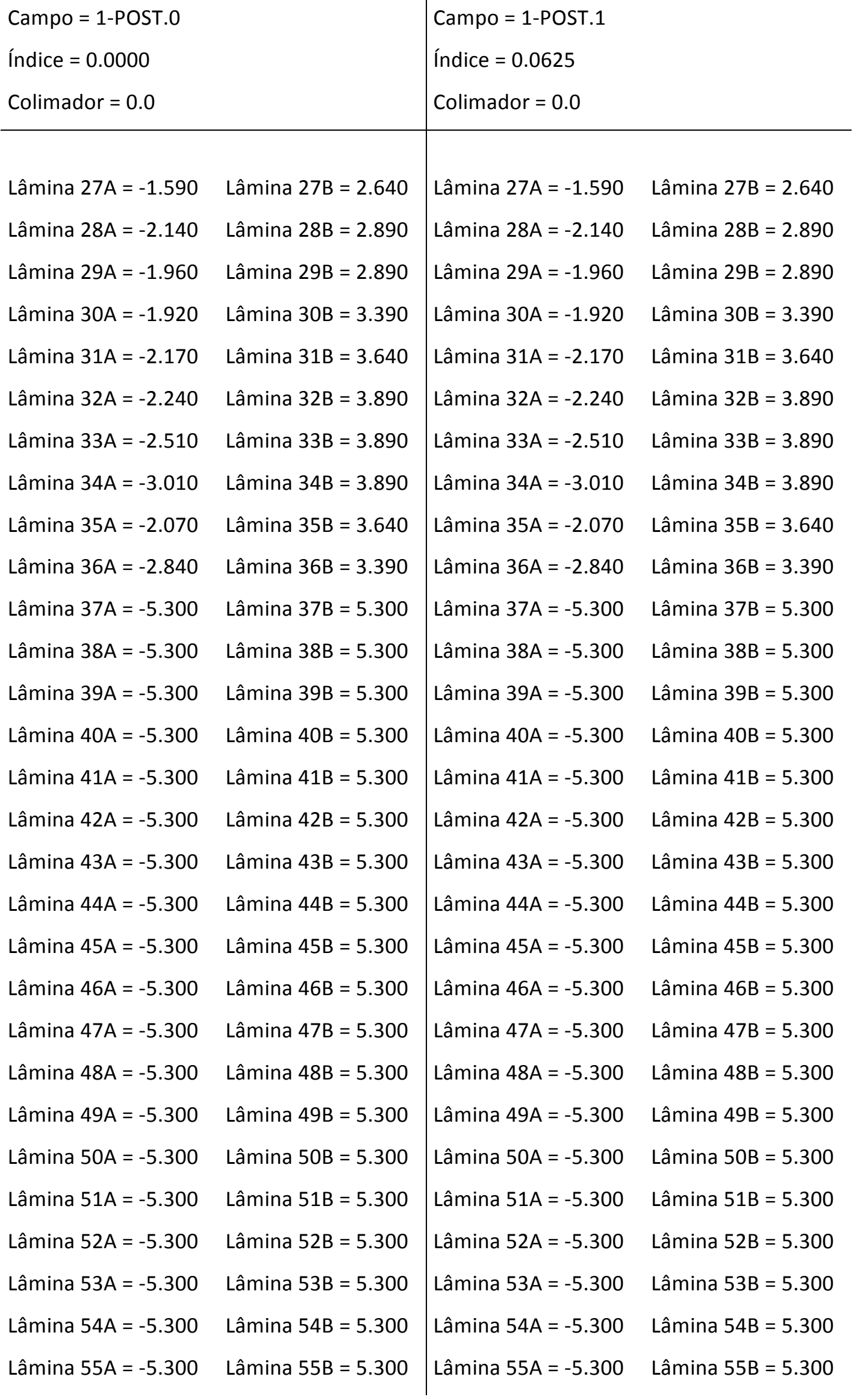




\begin{tabular}{|c|c|c|c|}
\hline \multicolumn{2}{|l|}{ Campo = 1-POST .0} & \multicolumn{2}{|l|}{ Campo = 1-POST.1 } \\
\hline Índice = 0.0000 & & Índice $=0.0625$ & \\
\hline Colimador $=0.0$ & & Colimador $=0.0$ & \\
\hline Lâmina $56 \mathrm{~A}=-5.300$ & Lâmina 56B = 5.300 & Lâmina $56 \mathrm{~A}=-5.300$ & Lâmina 56B = 5.300 \\
\hline Lâmina 57A $=-5.300$ & Lâmina 57B = 5.300 & Lâmina 57A $=-5.300$ & Lâmina 57B $=5.300$ \\
\hline Lâmina $58 \mathrm{~A}=-5.300$ & Lâmina 58B = 5.300 & Lâmina $58 \mathrm{~A}=-5.300$ & Lâmina 58B = 5.300 \\
\hline Lâmina 59A $=-5.300$ & Lâmina 59B = 5.300 & Lâmina 59A $=-5.300$ & Lâmina 59B = 5.300 \\
\hline Lâmina $60 \mathrm{~A}=-5.300$ & Lâmina 60B = 5.300 & Lâmina $60 \mathrm{~A}=-5.300$ & Lâmina 60B $=5.300$ \\
\hline Nota $=0$ & & Nota $=0$ & \\
\hline Formato $=0$ & & Formato $=0$ & \\
\hline Magnificação = 1.00 & & Magnificação = 1.00 & \\
\hline
\end{tabular}




\section{REFERÊNCIAS BIBLIOGRÁFICAS}

Ahnesjö A, Knoos T, Montelius A. "Application of the convolution method for calculation of output factors for therapy photon beams." (1992) Med. Phys. 19, 295-302.

Ahnesjö A. "Analytic modeling of photon scatter from flattening filters in photon therapy beams," (1994) Med. Phys. 21, 1227-1235.

Ahnesjö A, Aspradakis, M M. "Dose calculations for external photon beams in radiotherapy” (1999) Phys. Med. Biol. 44, 99-155.

Andreo P, Burns D T, Hohlfeld K, Huq M S, Kanai T, Laitano F, Smyth V, Vynckier S. "Absorbed dose determination in external beam radiotherapy: an international Code of Practice for dosimetry based on standards of absorbed dose to water. (2000) Technical Reports Series 398”. Vienna, Austria, IAEA.

Bakai A, Alber M, Nusslin F. "A revision of the gamma-evaluation concept for the comparison of dose distributions". (2003) Phys. Med. Biol. 48, 3543-3553.

Bayouth J E, Wendt D, Morrill S M, "MLC quality assurance techniques for IMRT applications". (2003) Med. Phys. 30, 742-750.

Bortfeld T R, Kahler D L, Waldron T J, Boyer A L. "X-ray field compensation with multileaf collimators," (1994) Int. J. Rad. Oncol. Biol. Phys. 28, 723 - 730.

Boyer A, Biggs P, Galvin J, Klein E, Losasso T, Low D, Mah K, Yu C. "Basic applications of multileaf collimators: Report of AAPM Radiation Therapy Committee Task Group 50”. (2001a) Report 72. Med. Phys.

Boyer A, Butler E B, DiPetrillo T A, Engler M J, Fraass B, Grant W, Ling C C, Low D A, Mackie T R, Mohan R, Purdy J A, Roach M, Rosenman J G, Verhey L J, Wong J W, Cumberlin R L, Stone H, Palta J R. "Intensity Modulated Radiotherapy: Current status and issues of interest" (2001b) Int. J. Rad. Oncol. Biol. Phys. 51, 880 - 914.

Boyer A, Xing L, Ma C M, et al. "Theoretical considerations of monitor unit calculations for intensity modulated beam treatment planning". (1999) Med. Phys. 26, 187-195.

Brahme, A. "Design principles and clinical possibilities with a new generation of radiation therapy equipment: a review," (1987) Acta Oncol. 26, 403-412.

Brahme, A. "Optimization of stationary and moving beam radiation therapy techniques" (1988) Rad. Oncol. 12, 129-140.

Chui C S, Chan M F, Yorke E, Spirou S, Ling C C, “'Delivery of intensity-modulated radiation therapy with a conventional multileaf collimator: Comparison of dynamic and segmental methods"' (2001) Med. Phys. 28, 2441-2449.

Clarkson J R. " A note on depth doses in the fields of irregular shape" (1941) Br. J. Rad. $14,265-268$.

Convery D J, Rosenbloom M E. "The generation of intensity-modulated fields for conformal radiotherapy by dynamic collimation” (1992) Phys. Med. Biol. 37, 13591374. 
Cormack A M. “A problem in rotation therapy with x rays" (1987) Int. J. Rad. Oncol. Biol. Phys. 13, $623-630$.

Day M J, Aird G A. "The equivalent-field method for dose determinations in rectangular fields" (1983) Br. J. Rad. (Suppl.) 17, 105-114.

Dirkx M L P, Heijmen B J M, Santvoort J P C. "Leaf trajectory calculation for dynamic multi-leaf collimation to realize optimized fluence profiles" (1998) Phys. Med. Biol. $43,1171-1184$.

ESTRO (1997): European Society for Therapeutic Radiology and Oncology. Monitor Unit Calculation for High Energy Photon Beams. Physics for Clinical Radiotherapy, Booklet $n^{\circ} 3$. ESTRO.

Ezzell G A, Galvin J M, Low D, Palta J R, Rosen I, Sharpe M B, Xia P, Xiao Y, Xing L and Yu C X. "Guidance document on delivery, treatment planning, and clinical implementation of IMRT: report of the IMRT Subcommittee of the AAPM Radiation Therapy Committee" (2003) Med. Phys. 30, 2089-115

Fraass B, Doppke K, Hunt M, Kutcher G, Starkschall G, Stern R and Van Dyke J "American Association of Physicists in Medicine Radiation Therapy Committee Task Group 53: quality assurance for clinical radiotherapy treatment planning" (1998) Med. Phys. 25, 1773-829.

Graves M N, Thompson A V, Martel M K, McShan D L, Fraass B A. "Calibration and quality assurance for rounded leaf-end MLC systems” (2001) Med. Phys. 28, 22272233.

Hardcastle N, Metcalfe P, Ceylan A, Williams M J. "Multileaf collimator end leaf leakage: Implications for wide-field IMRT” (2007) Phys. Med. Biol. 52, 493 - 504.

IAEA, Technical Reports Series No. 398 (2000). Absorbed dose determination in external beam radiotherapy. An international code of practice for dosimetry based on standards of absorbed dose to water.

ICRU (1976): International Commission on Radiation Units. Determination of absorbed dose in a patient irradiated by beams of $\mathrm{X}$ or gamma rays in radiotherapy procedures. ICRU Report $n^{\circ} 24$.

ICRU (2010): International Commission on Radiation Units. Prescribing, recording, and reporting photon-beam intensity-modulated radiation therapy (IMRT). ICRU Report $\mathrm{n}^{\circ} 83$.

Jang S Y, Liu H H, Wang X, Vassiliev O N, Siebers J V, Dong L, Mohan R. "Dosimetric verification for intensity-modulated radiotherapy of thoracic cancers using experimental and Monte Carlo approaches" (2006) Int. J. Rad. Oncol. Biol. Phys. 66, $939-48$

Johansson K A, Horiot J C, Van Dam J, Lepinoy D, Sentenac I, Sernbo G. “Quality assurance control in the EORTC cooperative group of radiotherapy. Dosimetric intercomparison" (1986) Rad. Oncol. 7, 269-279.

Johns H E, Cunningham J R. The Physics of Radiology. (1984) Thomas, Springfield. 
Khan F M. The Physics of Radiation Therapy. (2003) 3rd edition, Lippincott Williams \& Wilkins.

Kung J H, Chen G T Y, Kuchnir F K. "A monitor unit verification calculation in intensity modulated radiotherapy as a dosimetry quality assurance" (2000) Med. Phys. 27(10), 2226-2230

Kutcher G J et al. "Comprehensive QA for radiation oncology: Report of AAPM Radiation Therapy Committee Task Group 40” (1994) Med. Phys. 21, 581-618

Liu H H, Mackie T R, McCullough E C. "A dual source photon beam model used in convolution/superposition dose calculations for clinical mega-voltage x-ray beams" (1997) Med. Phys. 24, 1960-1974.

Lorenz F, Killoran J H, Wenz F and Zygmanski P. "An independent dose calculation algorithm for MLC-based stereotactic radiotherapy" (2007) Med. Phys. 34, 1605-14

Low D A, Harms W B, Mutic S, Purdy J A, “A technique for the quantitative evaluation of dose distributions" (1998) Med. Phys. 25, 656-661.

Low D A, Dempsey J F. "Evaluation of the gamma dose distribution comparison method" (2003) Med. Phys. 30, 2455-2464.

Low D A, Moran J M, Dempsey J F, Dong L, Oldham M. "Dosimetry tools and techniques for IMRT" (2011) Med. Phys. 38(3): 1313-38.

Podgorsak E B. Radiation Oncology Physics: a Handbook for Teachers and Students (2005) Vienna, Austria. AIEA

Rogers D W O, Faddegon B A, Ding G X, Ma C M, Wei J, Mackie T R. "BEAM: A Monte Carlo code to simulate radiotherapy treatment units" (1995) Med. Phys. 22, 503-524.

Sharpe M B, Jaffray D A, Battista J J, Munro P. "Extrafocal radiation: A unified approach to the prediction of beam penumbra and output factors for megavoltage x-ray beams" (1995) Med. Phys. 22: 2065-2074.

Sievinen J, Ulmers W, Kaissl W. "AAA photon dose calculation model in eclipse" (2005) Varian RandD \#7170B. Palo Alto, CA: Varian Medical Systems.

Siochi R A C. "Minimizing static intensity modulation delivery time using an intensity solid paradigm" (1999) Int. J. Rad. Oncol. Biol. Phys. 43, 671-680.

Spirou S V, Chui C S. "Generation of arbitrary intensity profiles by dynamic jaws or multileaf collimators” (1994) Med. Phys. 21, 1031-1041.

Stern R L et al. "Verification of monitor unit calculations for non-IMRT clinical radiotherapy: Report of AAPM Radiation Therapy Committee Task Group 114." (2011) Med. Phys. 38, 504-529.

Stock M, Kroupa B, Georg D. "Interpretation and evaluation of the gamma index and the gamma index angle for the verification of IMRT hybrid plans" (2005) Phys. Med. Biol. 50, 399-411. 
Storchi P R M, van Battum L J, Woudstra E. "Calculation of a pencil beam kernel form measured photon beam data" (1999) Phys. Med. Biol. 44, 2917-2928.

Van Dyk J, Barnett R B, Cygler J E, Shragge P C. "Commissioning and quality assurance of treatment planning computers" (1993) Int. J. Rad. Oncol. Biol. Phys. 26, 261-273.

Xing L, Chen Y, Luxton G, Li J G, Boyer A L. "Monitor unit calculation for an intensity modulated photon field by a simple scatter - summation algorithm" (2000) Phys. Med. Biol. 45, N1-N7.

Yu C X. "Intensity-modulated arc therapy with dynamic multileaf collimation: An alternative to tomotherapy" (1995) Phys. Med. Biol. 40, 1435-1449.

Zhu J, Yin F F, Kim J H. "Point dose verification for intensity modulated radiosurgery using Clarkson's method” (2003) Med. Phys. 30, 2218 - 2221. 